\title{
Impact of Quantized Inter-agent Communications on Game-Theoretic and Distributed Optimization Algorithms
}

\author{
Ehsan Nekouei, Tansu Alpcan, Robin J. Evans
}

\begin{abstract}
Quantized inter-agent communications in game-theoretic and distributed optimization algorithms generate uncertainty that affects the asymptotic and transient behavior of such algorithms. This chapter uses the information-theoretic notion of differential entropy power to establish universal bounds on the maximum exponential convergence rates of primal-dual and gradient-based Nash seeking algorithms under quantized communications. These bounds depend on the inter-agent data rate and the local behavior of the agents' objective functions, and are independent of the quantizer structure. The presented results provide trade-offs between the speed of exponential convergence, the agents' objective functions, the communication bit rates, and the number of agents and constraints. For the proposed Nash seeking algorithm, the transient performance is studied and an upper bound on the average time required to settle inside a specified ball around the Nash equilibrium is derived under uniform quantization. Furthermore, an upper bound on the probability that the agents' actions lie outside this ball is established. This bound decays double-exponentially with time.
\end{abstract}

\section{Introduction}

Modern societies are heavily dependent on networking technologies for almost every type of activity. The Internet, smart phones, and cloud computing could not exist without networking. In all networked systems, a limited number of resources, e.g., bandwidth and computing power, are shared among the interconnected devices, hereafter called the agents. The performance of the networked system is highly dependent on how these resources are shared among the agents. Hence, resource allocation algorithms play an important role in networking technologies. The network resource allocation problem between the agents can be formulated as a global optimization problem with a team-optimal solution, or modeled as a non-cooperative game. The solution in the former case is team-optimal, whereas the resources are shared according to the equilibrium of the game among the selfish agents in the latter.

Ehsan Nekouei

KTH Royal Institute of Technology, Osquldas väg 10, KTH Main Campus, stockholm, Sweden, e-mail: nekouei@kth.se

Tansu Alpcan

The University of Melbourne, Parkville, Melbourne Australia, Address of Institute e-mail: tansu.alpcan@ unimelb.edu.au

Robin J. Evans

The University of Melbourne, Parkville, Melbourne Australia, Address of Institute e-mail: robinje@ unimelb.edu.au 
This chapter studies two distinct scenarios for the network resource sharing problem related to these cases. In the first scenario, the resource allocation problem is posed as a network utility maximization (NUM) problem, and the agents deploy a distributed, iterative primal-dual optimization algorithm to solve the NUM problem. In the second scenario, the interaction between the agents is modeled as a non-cooperative game and the agents compute the Nash equilibrium (NE) solution of the game using a gradient-based Nash seeking algorithm. The communication channels between the agents are modeled as digital ones since the agents may be far away from each other. The actions of the agents are hence quantized into discrete-valued symbols that may are represented as bits. The finite capacity of practical communication leads to an upper bound on the average number of bits transmitted per unit time. Consequently, the agents' local variables can only be transmitted in a quantized form using a finite number of bits per time interval. It is known in the literature that such data rate limitations can have detrimental impacts on the performance of control and optimization algorithms. For example, a communication channel deployed in a feedback control system can destabilize the system if its data rate is too low, e.g., see [1] and [2]. Moreover, in distributed optimization as well as Nash equilibrium seeking algorithms, the quantized communications results in information ambiguity since each agent receives only quantized information from the other agents (which is typically different from unquantized information).

The aim of this chapter is to quantify the impact of quantized communications in NUM problems and non-cooperative games by making use of information-theoretic ideas. The results presented integrate and summarize those in [3] and [4].

The remainder of the chapter is organized as follows. The next section introduces the quantized primaldual algorithm for NUMs and studies its asymptotic performance under quantized inter-agent communications. In section 3, a quantized gradient-based Nash seeking algorithm is proposed and its asymptotic and non-asymptotic behaviors are analyzed under quantized communications. Section 3.3 presents the numerical results, which is followed by the concluding remarks of the Section 4 .

\section{Primal-dual Algorithm Under Quantized Communications}

In the seminal work [5], Kelly et al. introduced the network utility maximization (NUM) approach, which provides decentralized frameworks in the form of primal, dual, and primal-dual (PD) decomposition methods, for solving network resource allocation problems. Each decomposition method distributes the computational burden of solving the resource allocation problem among the agents, while the task of information transfer between the agents is handled by the underlying communication network. The problem of devising efficient decomposition methods for NUM problems has been extensively studied in the literature, e.g., see [6] and the references therein. While the performance of distributed optimization algorithms, in particular of NUM algorithms, is well understood under perfect communication channels, investigation of the impact of imperfect communications on these optimization algorithms is relatively a new research area that has attracted much interests in the recent years, e.g., see [7], [8].

This section focuses on a NUM problem in which a group of agents maximize the sum of their local concave objective functions subject to a set of linear constraints using a quantized PD algorithm with a random initial condition. Following the conventions of the NUM literature, e.g., see [5], [6] and the references therein, it is assumed that the primal variables are updated by the agents, whereas each dual variable is updated by a network node (NN) that has access to the knowledge of the the constraint associated with that specific dual variable. Thus, the agents and NNs need to exchange the quantized values of the primal and 
dual variables to execute the PD algorithm. The impact of quantized communications between the agents and NNs on the convergence rate of the PD algorithm under quantization is investigated in this setup.

The rest of this section first studies the system model, and then describes the communication graph, the structure of quantizers, as well as the underlying standing assumptions. Asymptotic and non-asymptotic results on the convergence of PD algorithm under quantization are presented.

\subsection{NUM Model}

A specific formulation of the NUM problem as one of convex optimization involves $M$ agents, who maximize the sum of their individual objective functions subject to a set of linear equality constraints. Let $x^{i}$ and $U_{i}\left(x^{i}\right)$ represent the decision variable of the agent $i$ and its objective function, respectively. Assume that the objective function of each agent is concave in its decision variable. The agents then collectively solve the following NUM problem:

$$
\begin{aligned}
& \underset{x}{\operatorname{maximize}} \sum_{i}^{M} U_{i}\left(x^{i}\right), \\
& \text { Subject to } A x=b \text {, }
\end{aligned}
$$

where, $M$ is the number of agents, $b \in \mathbb{R}^{N}, A \in \mathbb{R}^{N \times M}, N$ is the number of constraints, and $x=\left[x^{1}, \cdots, x^{M}\right]^{\top}$. The condition $N<M$ is imposed to ensure that the feasible set of the optimization problem (1) is nonempty. The matrix $A$ is assumed to be full rank, i.e., $\operatorname{rank}(A)=N$, to ensure the uniqueness of the dual optimal solution. The objective function in (1) is concave and the constraints are linear. Thus, the centralized optimization problem (1) can be solved using the standard convex optimization techniques.

When solving the problem in a distributed manner using the PD algorithm [6], the primal and dual variables are updated according to

$$
\begin{aligned}
& x_{k}^{i}=x_{k-1}^{i}+\mu_{k-1}\left(\frac{d}{d x^{i}} U_{i}\left(x_{k-1}^{i}\right)-A_{i}^{\top} \lambda_{k-1}\right), 1 \leq i \leq M \\
& \lambda_{k}^{j}=\lambda_{k-1}^{j}+\mu_{k-1}\left(\bar{A}_{j} x_{k-1}-b_{j}\right) \quad 1 \leq j \leq N,
\end{aligned}
$$

where $\mu_{k-1}$ is the step size of the algorithm at iteration $k-1, x_{k}^{i}$ and $\lambda_{k}^{j}$ denote the values of $i$ th primal variable and $j$ th dual variable at iteration $k$, respectively. In terms of notation, $\lambda_{k-1}=\left[\lambda_{k-1}^{1}, \cdots, \lambda_{k-1}^{N}\right]^{\top}$, $A_{i}$ denotes the $i$ th column of the matrix $A$, and $\bar{A}_{j}$ denotes the $j$ th row of matrix $A$. The solution of the optimization problem (1) is obtained following a primal-dual (PD) decomposition approach in which the primal variables, i.e., agents' decision variables, are updated by the agents at each iteration. In addition, at each iteration of the PD algorithm, the $j$ th dual variable, i.e., $\lambda^{j}$, is updated by the specific $j$ th network node $(\mathrm{NN})$ with knowledge of the parameters characterizing the constraint associated with $\lambda^{j}$, i.e., $A_{j}$ and $b_{j}$. The vector of PD variables at iteration $k$, i.e., $y_{k}$, is defined as the vector concatenation of $x_{k}$ and $\lambda_{k}$, i.e.,

$$
y_{k}=\left[x_{k}, \lambda_{k}\right] .
$$

It is assumed that the initial primal and dual variables, i.e., $x_{0}$ and $\lambda_{0}$, are chosen randomly according to the probability density functions $p_{x_{0}}(x)$ and $p_{\lambda_{0}}(\lambda)$, respectively. By allowing the initial condition to be random, the primal and dual variables become random variables. This facilitates the use information theo- 
retic tools for studying the speed of exponential convergence of the primal-dual algorithm under quantized communications. Furthermore, the following assumptions are imposed on the objective functions of agents, the step size $\mu_{k}, p_{x_{0}}(x)$, and $p_{\lambda_{0}}(\lambda)$.

1. The agents' objective functions are concave and twice continuously differentiable.

2. $U_{i}^{\min } \leq \frac{d^{2}}{d x^{i^{2}}} U_{i}\left(x^{i}\right) \leq U_{i}^{\max }<0$ for $x_{i} \in \mathbb{R}$ and all $i$.

3. $\mu_{k} \leq \min _{i} \frac{1}{\left|U_{i}^{\min }\right|}$ for all $k$.

4. The sequence $\left\{\mu_{k}\right\}_{k}$ converges to $\mu^{\star}>0$.

5. The random vectors $x_{0}$ and $\lambda_{0}$ are mutually independent and the distributions of $x_{0}$ and $\lambda_{0}$ have finite differential entropies, i.e.,

$$
\begin{array}{r}
\left|-\int p_{x_{0}}(x) \log \left(p_{x_{0}}(x)\right) d x\right|<\infty \\
\left|-\int p_{\lambda_{0}}(\lambda) \log \left(p_{\lambda_{0}}(\lambda)\right) d \lambda\right|<\infty
\end{array}
$$

Assumptions 1 and 2 above are standard in the optimization literature. Assumption 2 implies that the objective functions of agents are strongly concave and the first derivative of each objective function is Lipschitz continuous. Assumption 4 implies that the unquantized update rule does not employ a diminishing step-size rule as the PD update may not converge exponentially with such a step-size rule. Assumptions 3 and 4 , which are not commonly used in the literature, allow usage of the entropy power method from information theory. Assumption 5 implies that the initial condition injects a minimum amount of uncertainty to the PD algorithm, and the amount of uncertainty due to the initial condition is bounded. Variants of assumption 5 are used in the quantized feedback control literature [1].

\subsubsection{Communication Topology and Cost}

The inter-agent communication topology is represented as a bipartite graph induced by the $N \times M$ constraint matrix $A$. In this graph, the edges exist only between the agents and the network nodes (NNs), which form two disjoint sets of vertices. There exists an edge between agent $i$ and $\mathrm{NN} j$ in the communication graph if and only if $A_{j i} \neq 0$.

The communication mechanism is broadcast in nature, with each vertex 'listening' and broadcasting only to those other vertices with which it shares an edge. This is implemented by uniquely assigning every vertex in the graph one of $N+M$ disjoint transmission radio-frequency bands (frequency division multiplexing) or one of $N+M$ disjoint time slots per cycle (time division multiplexing), before the system is deployed. Any other vertex that needs to listen to a transmission just tunes in to the appropriate frequency band or time slot dedicated to the corresponding transmitter. Note that the edges do not represent individual one-to-one channels, but indicate the broadcast transmitter-receiver structure of the system.

Under typical digital modulation formats, the width of the frequency band/time-slot allocated to agent $i$ and/or the average transmission power it consumes to broadcast its encoded symbols to all NNs $j$ with $A_{j i} \neq 0$ will be proportional to its average data rate $R_{x}^{i}:=\lim _{k \rightarrow \infty} \frac{1}{k} \sum_{t=0}^{k-1} \log \left|\mathscr{A}_{i, t}^{x}\right|$. Similarly, the band/slot-width and/or transmission power used by NN $j$ to broadcast its encoded dual symbols to all agents $i$ with $A_{j i} \neq 0$ is typically proportional to $R_{\lambda}^{j}:=\lim _{k \rightarrow \infty} \frac{1}{k} \sum_{t=0}^{k-1} \log \left|\mathscr{A}_{j, t}^{\lambda}\right|$. Equation (5) in the upcoming section, which can be intuitively interpreted as $\sum_{i=1}^{M} R_{x}^{i}+\sum_{j=1}^{N} R_{\lambda}^{j}$, then captures the total amount of physical resources, i.e., 
time, bandwidth, or transmission power, required for the system to communicate. It can be seen that this communication cost scales with $O(N+M)$ as the network grows in size. Note that due to the broadcast nature of the system, every transmission can be heard by multiple receivers, without the transmitter having to use up extra resources.

\subsubsection{A Quantizer Structure for NUM}

To execute the PD update rule (2), the agents and NNs require the knowledge of dual and primal variables, respectively. Since the agents and NNs are not necessarily co-located, the information exchange between NNs and agents is performed via broadcast communication channels, as described in the next subsection. Due to the capacity limitations of these channels, only quantized versions of the primal and dual variables can be exchanged between NNs and agents.

At iteration $k$, agent $i$ encodes $x_{k}^{i}$ to $\hat{Q}_{i, k}^{x}$ using an adaptive encoder mapping of the form

$$
\hat{Q}_{i, k}^{x}=E_{i, k}^{x}\left(\left\{x_{n}^{i}\right\}_{n=0}^{k},\left\{\hat{Q}_{i, n}^{x}\right\}_{n=0}^{k-1}\right) .
$$

It then broadcasts $\hat{Q}_{i, k}^{x}$ to all NNs $j$ with $A_{j i} \neq 0$. The output of the encoder of agent $i$ at iteration $k$, i.e., $\hat{Q}_{i, k}^{x}$, belongs to the finite alphabet set $\mathscr{A}_{i, k}^{x}$. Thus, agent $i$ requires $\log _{2}\left|\mathscr{A}_{i, k}^{x}\right|$ bits to transmit its encoded symbol to NNs. A large value of $\left|\mathscr{A}_{i, k}^{x}\right|$ indicates that agent $i$ transmits its decision variable with high precision to NNs whereas a low $\left|\mathscr{A}_{i, t}^{x}\right|$ indicates low quality communication between agent $i$ and NNs. Upon receiving $\hat{Q}_{i, k}^{x}$, all NNs $j$ with $A_{j i} \neq 0$ reconstruct the quantized estimate of $x_{k}^{i}$, i.e., $Q_{i, k}^{x}$, using the decoder mapping $Q_{i, k}^{x}=D_{i, k}^{x}\left(\left\{\hat{Q}_{i, n}^{x}\right\}_{n=0}^{k}\right)$.

Similarly, at iteration $k$, NN $j$ chooses symbol $\hat{Q}_{j, k}^{\lambda}$ from the finite alphabet set $\mathscr{A}_{j, k}^{\lambda}$ according to the adaptive encoding map

$$
\hat{Q}_{j, k}^{\lambda}=E_{k}^{\lambda}\left(\left\{\lambda_{n}^{j}\right\}_{n=0}^{k},\left\{\hat{Q}_{j, n}^{\lambda}\right\}_{n=0}^{k-1}\right)
$$

and broadcasts $\hat{Q}_{j, k}^{\lambda}$ to all the agents with index $i$, where $A_{j i} \neq 0$. Next, all agents $i$ with $A_{j i} \neq 0$ construct the quantized version of $\lambda_{k}^{j}$, i.e., $Q_{j, k}^{\lambda}$, using the decoding map $Q_{j, k}^{\lambda}=D_{j, k}^{\lambda}\left(\left\{\hat{Q}_{j, n}^{\lambda}\right\}_{n=0}^{k}\right)$. Note that this formulation allows the encoded symbol at iteration $k$ to depend on the current and past values of the primal/dual variables as well as the past outputs of the encoder.

Let $\mathscr{Q}=\left\{\left\{E_{i, k}^{x}(\cdot), D_{i, k}^{x}(\cdot)\right\}_{i},\left\{E_{j, k}^{\lambda}(\cdot), D_{j, k}^{\lambda}(\cdot)\right\}_{j}\right\}_{k=0}^{\infty}$ be a quantization scheme. Then, the quantized versions of the PD variables at iteration $k$ under the quantization scheme $\mathscr{Q}$ are denoted by $Q_{k}$, i.e.,

$$
Q_{k}=\left[Q_{k}^{x}, Q_{k}^{\lambda}\right]
$$

where $Q_{k}^{x}=\left[Q_{1, k}^{x}, \cdots, Q_{M, k}^{x}\right]^{\top}$ and $Q_{k}^{\lambda}=\left[Q_{1, k}^{\lambda}, \cdots, Q_{N, k}^{\lambda}\right]^{\top}$. 
Next, three notions of data rate for a given quantization scheme $\mathscr{Q}$ are defined and later used to study the convergence behavior of primal, dual, and PD variables. The average aggregate data rate per unit time for transmitting the primal variables to NNs under the quantization scheme $\mathscr{Q}, R_{x}$, is defined as

$$
R_{x}=\lim _{k \rightarrow \infty} \frac{1}{k} \sum_{t=0}^{k-1}\left(\sum_{i=1}^{M} \log \left|\mathscr{A}_{i, t}^{x}\right|\right)
$$

Similarly, define the average aggregate data rate per unit time for broadcasting the dual variables to agents under the quantization scheme $\mathscr{Q}, R_{\lambda}$, as

$$
R_{\lambda}=\lim _{k \rightarrow \infty} \frac{1}{k} \sum_{t=0}^{k-1}\left(\sum_{j=1}^{N} \log \left|\mathscr{A}_{j, t}^{\lambda}\right|\right)
$$

Finally, the average total date rate per unit time under the quantization scheme $\mathscr{Q}$, i.e., $R_{\mathscr{Q}}$, is defined as

$$
R_{\mathscr{Q}}=\lim _{k \rightarrow \infty} \frac{1}{k} \sum_{t=0}^{k-1}\left(\left(\sum_{i=1}^{M} \log \left|\mathscr{A}_{i, t}^{x}\right|\right)+\sum_{j=1}^{N} \log \left|\mathscr{A}_{j, t}^{\lambda}\right|\right)
$$

The quantized PD update rule under the quantization scheme $\mathscr{Q}$ is formulated as

$$
\begin{aligned}
& x_{k}^{i}=x_{k-1}^{i}+\mu_{k-1}\left(\frac{d}{d x^{i}} U_{i}\left(x_{k-1}^{i}\right)-A_{i}^{\top} Q_{k-1}^{\lambda}\right), \\
& \lambda_{k}^{j}=\lambda_{k-1}^{j}+\mu_{k-1}\left(\bar{A}_{j} Q_{k-1}^{x}-b_{j}\right)
\end{aligned}
$$

Let $x^{\star}, \lambda^{\star}$ be the primal optimal and dual optimal solutions, respectively. Further, let $y^{\star}$ be the vector concatenation of $x^{\star}, \lambda^{\star}$. Define $\varepsilon_{k}=y_{k}-y^{\star}$ as the difference between the PD variables at iteration $k$ and the optimal solution. Let $\left\|\varepsilon_{k}\right\|_{2}$ denote the distance of the $\mathrm{PD}$ variables at iteration $k$ from optimal solution, i.e.,

$$
\left\|\varepsilon_{k}\right\|_{2}=\sqrt{\sum_{i=1}^{M}\left(x_{k}^{i}-x^{i \star}\right)^{2}+\sum_{j=1}^{N}\left(\lambda_{k}^{j}-\lambda j^{\star}\right)^{2}},
$$

where $x^{i \star}$ and $\lambda^{j^{\star}}$ are the optimal values of the primal variable $x^{i}$ and the dual variable $\lambda^{j}$, respectively. Then, the mean square distance (MSD) of the PD variables from the optimal solution at iteration $k$ under the quantization scheme $\mathscr{Q}$ is defined as $\mathrm{E}\left[\left\|\varepsilon_{k}\right\|_{2}^{2}\right]$. Define the MSD of the primal variables from the optimal primal solution at iteration $k$ as $\mathrm{E}\left[\left\|\varepsilon_{k}^{x}\right\|_{2}^{2}\right]$ where $\varepsilon_{k}^{x}=x_{k}-x^{\star}$. Similarly, the MSD of the dual variables at iteration $k$ from the optimal dual solution is defined as $\mathrm{E}\left[\left\|\varepsilon_{k}^{\lambda}\right\|_{2}^{2}\right]$ where $\varepsilon_{k}^{\lambda}=\lambda_{k}-\lambda^{\star}$. Next, the class of optimum achieving (OA) quantization schemes are defined.

Definition 1. The quantization scheme $\mathscr{Q}$ is called an OA quantization scheme if, under $\mathscr{Q}$, the primal and dual variables converge to their optimal values $x^{\star}$ and $\lambda^{\star}$. That is:

$$
\begin{gathered}
\lim _{k \rightarrow \infty} x_{k}=x^{\star} \\
\lim _{k \rightarrow \infty} \lambda_{k}=\lambda^{\star}
\end{gathered}
$$


Definition 1 implies that, under an OA quantization scheme, the quantization error does not impede the convergence of the PD algorithm to the optimal solution. Thus, under an OA quantization scheme, the PD algorithm converges to the optimal solution of the optimization problem regardless of the quantized communication between agents and NNs.

\subsection{Distributed Optimization Results and Discussion}

This section analyzes the impact of quantized communications on the mean square distance (MSD) from the optimal solution of the primal and dual variables generated by the primal-dual algorithm (PD) for two different regimes: $(i)$ Asymptotic regime, (ii) Non-asymptotic regime. In the asymptotic regime, the behavior of the MSD under OA quantization schemes is studied as the number of iterations $k$ increase to infinity. To this end, the notion of distance decay exponent (DDE) is introduced, which captures the rate of exponential convergence of the MSD to zero. Universal lower bounds on the DDE of PD variables, namely the primal variables and dual variables, are established in Theorems 1, 2, 3, and 4. In the non-asymptotic regime, the behavior of the MSD is investigated for any finite $k$. Here, the results provide universal lower bounds on the MSD for any finite $k$ (see Corollaries 1 and 2 for more details).

\subsubsection{Asymptotic behavior of PD algorithm under Quantization}

This subsection first introduces the notion of the distance decay exponent (DDE) for the primal and dual variables in PD. Subsequently, universal lower bounds on the DDE of PD primal and dual variables are derived.

Definition 2. Let $\mathscr{Q}$ be an OA quantization scheme. Then, the DDE of the PD primal and dual variables under $\mathscr{Q}$ are defined as

$$
\begin{gathered}
\liminf _{k \rightarrow \infty} \frac{1}{k} \log \mathrm{E}\left[\left\|\varepsilon_{k}\right\|_{2}^{2}\right], \\
\underset{k \rightarrow \infty}{\liminf } \frac{1}{k} \log \mathrm{E}\left[\left\|\varepsilon_{k}^{x}\right\|_{2}^{2}\right], \\
\liminf _{k \rightarrow \infty} \frac{1}{k} \log \mathrm{E}\left[\left\|\varepsilon_{k}^{\lambda}\right\|_{2}^{2}\right],
\end{gathered}
$$

respectively.

The DDEs capture the speed of exponential mean square convergence of the PD primal and dual variables to their corresponding optimal solutions. These are non-positive quantities, where a more negative DDE indicates faster convergence to the optimal solution. Moreover, a zero DDE implies slower-than-exponential convergence. In this subsection, the information-theoretic notion of entropy power is used to establish universal lower bounds on the DDE of the primal and dual variables.

The next theorem provides a universal lower bound on the DDE of the PD variables under OA quantization schemes. The proof uses the information-theoretic notion of differential entropy power, which has been previously applied to study control with communication constraints; see e.g. [9]. 
Theorem 1. Let $\mathscr{Q}$ be an $O A$ quantization scheme. Then, the DDE of PD variables under $\mathscr{Q}$ can be bounded from below

$$
\liminf _{k \rightarrow \infty} \frac{1}{k} \log \mathrm{E}\left[\left\|\varepsilon_{k}\right\|_{2}^{2}\right] \geq \frac{2}{N+M}\left(\sum_{i=1}^{M} \log \left(1+\mu^{\star} \frac{d^{2}}{d x^{i^{2}}} U_{i}\left(x^{i^{\star}}\right)\right)-R_{\mathscr{Q}}\right)
$$

where $x^{i^{\star}}$ is the optimal value of the primal variable $x^{i}$.

Proof. See Appendix.

Theorem 1 establishes an explicit universal lower bound on the DDE of PD variables under OA quantization schemes. This bound is universal in the sense that it is independent of the structure of the quantizer, and is thus applicable to all quantization schemes which are OA.

According to Theorem 1 , for a given average total data rate $R_{\mathscr{Q}}$, the $P D$ variables converge to the optimal solution at most exponentially fast. The speed of this exponential convergence is bounded by the average total data rate under the quantization scheme, i.e., $R_{\mathscr{Q}}$, and also by the behavior of the objective functions of agents around the optimal solution. As stated in Theorem 1, the lower bound on the DDE for PD variables decreases linearly with $R_{\mathscr{Q}}$. Note that as $R_{\mathscr{Q}}$ becomes large, the NNs and agents have more precise information about the primal and dual variables. The lower bound on the DDE also increases with the second derivatives of the agents' objective functions at the optimal solution. As these second derivatives becomes less negative, the objective function becomes flatter near the optimal solution and the quantized PD algorithm can be expected to converge more slowly. Theorem 1 is in concordance with this intuition.

The next theorem establishes a universal lower bound on the DDE of primal variables in the quantized PD update rule under an OA quantization scheme.

Theorem 2. [10] Under an OA quantization scheme $\mathscr{Q}$, the DDE of the primal variables is lower bounded by

$$
\liminf _{k \rightarrow \infty} \frac{1}{k} \log \mathrm{E}\left[\left\|\varepsilon_{k}^{x}\right\|_{2}^{2}\right] \geq \frac{2}{M}\left(\sum_{i=1}^{M} \log \left(1+\mu^{\star} \frac{d^{2}}{d x^{i^{2}}} U_{i}\left(x^{i^{\star}}\right)\right)-R_{\lambda}\right) .
$$

According to Theorem 2, the exponential convergence speed of the primal variables is limited by (i) the behavior of objective functions of the agents around the optimal solution, (ii) the average aggregate data rate for transmission of dual variables, and (iii) the number of agents. Different from the PD bound in Theorem 1), this lower bound on the DDE of the primal variables depends only on the average aggregate data rate for transmission of dual variables, i.e., $R_{\lambda}$, rather than on the average total data rate under the quantization scheme $\mathscr{Q}$. This observation signifies the role of the quantized dual variables on the convergence of the primal variables.

The next theorem presents a result on DDE for dual variables.

Theorem 3. [10] The DDE of dual variables under an OA quantization scheme $\mathscr{Q}$ satisfies

$$
\liminf _{k \rightarrow \infty} \frac{1}{k} \log \mathrm{E}\left[\left\|\varepsilon_{k}^{\lambda}\right\|_{2}^{2}\right] \geq-\frac{2}{N} R_{x} .
$$

Theorem 3 establishes a universal bound on the fastest possible exponential convergence rate of the dual variables under any $O A$ quantization scheme $\mathscr{Q}$. The lower bound in Theorem 3 is controlled by the number of constraints and the average aggregate data rate for transmission of primal variables to NNs. Compared 
to the PD lower bound, it does not depend on the behavior of the objective functions of agents and is only limited by the average aggregate data rate for transmission of the primal variables, i.e., $R_{x}$, rather than the average total data rate $R_{\mathscr{Q}}$.

Next, a lower bound on the DDE of the PD algorithm is derived for quadratic NUM problems under zoom-in quantization schemes (see Definition 3). This bound is tighter than the lower bound in Theorem 1 for the high data-rate regime. In a quadratic NUM problem, the objective function of agent $i$ is given by $U_{i}\left(x^{i}\right)=-\frac{a_{i}}{2}\left(x^{i}\right)^{2}+c_{i} x^{i}+f_{i}$ where $a_{i}$ is a positive constant. The unquantized PD algorithm for quadratic NUM problems can be written as

$$
\begin{aligned}
& x_{k}^{i}=\left(1-\mu a_{i}\right) x_{k-1}^{i}+\mu\left(c_{i}-A_{i}^{\top} \lambda_{k-1}\right), 1 \leq i \leq M \\
& \lambda_{k}^{j}=\lambda_{k-1}^{j}+\mu\left(\bar{A}_{j} x_{k-1}-b_{j}\right) \quad 1 \leq j \leq N
\end{aligned}
$$

Let $y_{k}$ be the vector concatenation of $x_{k}$ and $\lambda_{k}$. Then, (11) can be written as

$$
y_{k}=T y_{k-1}+\mu\left[\begin{array}{c}
c \\
-b
\end{array}\right]
$$

where $c=\left[c_{1} \cdots, c_{M}\right]^{\top}$ and the matrix $T$ is defined as

$$
T=\left[\begin{array}{cc}
\operatorname{Diag}\left(1-\mu a_{1}, \cdots, 1-\mu a_{M}\right) & -\mu A^{\top} \\
\mu A & I_{N}
\end{array}\right]
$$

in which $I_{N}$ denotes an $N$-by- $N$ identity matrix and $\operatorname{Diag}\left(1-\mu a_{1}, \cdots, 1-\mu a_{M}\right)$ is a diagonal matrix with the $i$ th diagonal element equal to $1-\mu a_{i}$.

Let $\tilde{Q}_{k}=\left\{\hat{Q}_{1, n}^{x}, \cdots, \hat{Q}_{M, n}^{x}, \hat{Q}_{1, n}^{\lambda}, \cdots, \hat{Q}_{N, n}^{\lambda}\right\}_{n=0}^{k}$ be the collection of encoders' outputs up to iteration $k$, respectively. The quantized $\mathrm{PD}$ update rule is

$$
\begin{aligned}
& x_{k}^{i}=T_{i i} x_{k-1}^{i}+\sum_{j=M+1}^{M+N} T_{i j} Q_{k}^{\lambda^{(j-M)}}+\mu c_{i} \\
& \lambda_{k}^{j}=\lambda_{k-1}^{j}+\sum_{i=1}^{M} T_{j i} Q_{k}^{x^{i}}-\mu b_{j}
\end{aligned}
$$

The quantized update rule is denoted by $y_{k+1}=\hat{T}\left(y_{k}, \tilde{q}_{k}\right)$ where $\tilde{q}_{k}$ is a realization of $\tilde{Q}_{k}$. We use $C_{k}\left(\tilde{q}_{k}\right)$ to represent the quantization cell corresponding to $\tilde{q}_{k}$, i.e., the set of points in $\mathbb{R}^{N+M}$ which are mapped to the same output by the encoder when $\tilde{Q}_{k}=\tilde{q}_{k}$. Next, a zoom-in quantization scheme is defined.

Definition 3. Consider the quantization scheme $\mathscr{Q}$, and let $C_{k}\left(\tilde{q}_{k}\right)$ be the quantization cell at iteration $k$ which contains $y_{k}$. Then, $\mathscr{Q}$ is a zoom-in quantization scheme if at time $k+1$ the image of $C_{k}\left(\tilde{q}_{k}\right)$ under $\hat{T}\left(\cdot, \tilde{q}_{k}\right)$ is quantized for all $k \in \mathbb{N}_{0}=\{0,1,2, \cdots\}$.

In addition to the assumptions stated in Section 2.1 we also require

1. The matrix $T$ is invertible and all its eigenvalues are inside the unit circle in the complex plane.

2. A zoom-in quantization scheme is employed and each primal/dual variable is independently quantized.

3. The distributions of initial primal and dual variables, i.e., $p_{x_{0}}(x)$ and $p_{\lambda_{0}}(\lambda)$, are bounded and have finite support sets. 
Fig. 1 Two dimensional lattice of integers $\mathbb{Z}^{2}(a)$ and the lattice $T \mathbb{Z}^{2}$ (b).
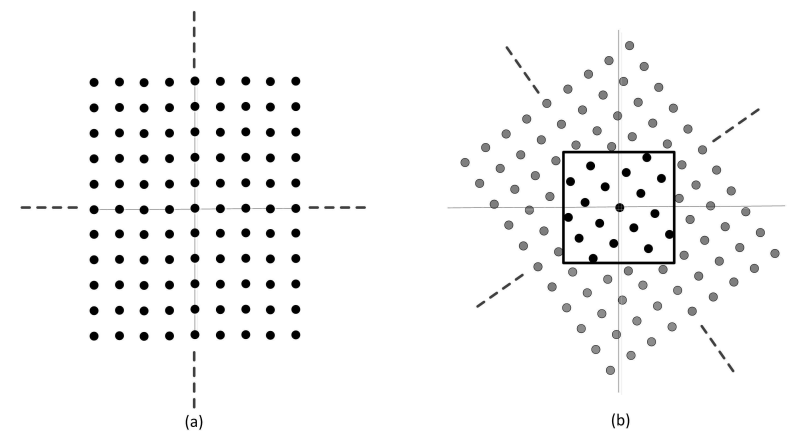

Theorem 4. [10] Consider any zoom-in quantization scheme $\mathscr{Q}$ with $\rho=\frac{\delta_{k}^{\max }}{\delta_{k}^{\min }}$ (for all $k$ ) where $\delta_{k}^{\max }$ and $\delta_{k}^{\min }$ are the maximum and minimum quantization steps under $\mathscr{Q}$ at iteration $k$, respectively. Let $\mathrm{B}$ be the hypercube centered at the origin with the ith side length equal to $4 \rho\left|T_{i i}\right|+2\|T\|_{\infty}$ where $\|\cdot\|_{\infty}$ denotes the norm infinity and $T_{i i}$ is the idiagonal entry of matrix $T$. Let $\beta_{T}$ be the number elements in the set $\mathrm{B} \cap T \mathbb{Z}^{N+M}$ where the lattice $T \mathbb{Z}^{N+M}$ is defined as $T \mathbb{Z}^{N+M}=\left\{T I, \quad I \in \mathbb{Z}^{N+M}\right\}$ and $\mathbb{Z}^{N+M}$ is the lattice of integers in $\mathbb{R}^{N+M}$. Then, the DDE of the PD variables under $\mathscr{Q}$ for quadratic NUM problems is lower bounded as

$$
\liminf _{k \rightarrow \infty} \frac{1}{k+1} \log \mathrm{E}\left[\left\|\varepsilon_{k+1}\right\|_{2}^{2}\right] \geq-\frac{2}{M+N} \log \left(\frac{\beta_{T}}{\left(\prod_{i=1}^{M+N}\left|T_{i i}\right|\right)}\right)
$$

Theorem 4 establishes a bound on the fastest possible exponential convergence speed of quantized PD algorithms in quadratic NUM problems, under any zoom-in quantization scheme which is OA. The lower bound in Theorem 4 depends on the number of agents, number of constrains and $\beta_{T}$. The constant $\beta_{T}$ depends on the dynamics of the unquantized PD algorithm, i.e., matrix $T$, and can be interpreted as the number of lattice points in $\mathbb{Z}^{N+M}$ which lie in B after applying the linear transformation $T$ to $\mathbb{Z}^{N+M}$. Fig. 1 shows the two dimensional lattice of integers $\mathbb{Z}^{2}$ and its image after applying a linear transformation. In Fig. $1(b)$, the number of lattice points in the square is equal to $\beta_{T}$. Since the transformation $T$ is linear, 0 always lies in $\mathrm{B}$ which implies $\beta_{T} \geq 1$.

Consider the PD algorithm in a quadratic NUM problem under the zoom-in quantization scheme $\mathscr{Q}$ with $\rho=\frac{\delta_{k}^{\max }}{\delta_{k}^{\min }}$. For the quadratic PD algorithms, Theorems 1 and 4 can be combined into

$$
\liminf _{k \rightarrow \infty} \frac{1}{k+1} \log \mathrm{E}\left[\left\|\varepsilon_{k+1}\right\|_{2}^{2}\right] \geq \frac{2}{M+N}\left(\left(\sum_{i=1}^{M} \log \left(1-\mu a_{i}\right)\right)-\min \left(\log \left(\beta_{T}\right), R_{\mathscr{Q}}\right)\right)
$$

If the quantization intervals for each primal/dual variable are divided into $K \geq 2$ equal length intervals, the data-rate under quantization scheme $\mathscr{Q}$ i.e., $R_{\mathscr{Q}}$, will increase by $(N+M) \log (K)$ bits and $\rho$ does not change. Hence, according to (14), the lower bound in Theorem 4 becomes tighter when compared to that in Theorem 1 as $R_{\mathscr{Q}}$ (or $K$ ) becomes large. This observation shows that the exponential convergence speed of the quantized PD algorithm in quadratic NUM problems cannot be made arbitrarily fast by increasing $R_{\mathscr{Q}}$. 
An upper bound on $\beta_{T}$ can be obtained by finding the number of lattice points of $\mathbb{Z}^{N+M}$ which lie in the smallest hypercube containing the image of B under $T^{-1}$. Let $T^{-1}(\mathrm{~B})$ be the image of the hypercube $\mathrm{B}$ under linear transformation $T^{-1}$. Let $\mathrm{B}_{T^{-1}}^{\star}$ be the smallest hypercube containing $T^{-1}(\mathrm{~B})$. Then, $\beta_{T}$ is upper bounded by $\prod_{i}\left(\left\lfloor l_{i}^{\star}\right\rfloor+1\right)$ where $l_{i}^{\star}$ is the $i$ th side length of $\mathrm{B}_{T^{-1}}^{\star}$. In the numerical analysis, this upper bound on $\beta_{T}$ is used to compute the lower bound in Theorem 4.

\subsubsection{PD algorithm in the non-asymptotic regime}

This subsection establishes universal lower bounds on the mean square distance (MSD) of primal-dual (PD), primal and dual variables from their corresponding optimal solutions at any finite time instance $k$. Unlike Theorems 1, 2 and 3, the following results are not limited to optimum achieving (OA) quantization schemes. Thus, they give rise to universal lower bounds on the MSD of PD, primal and dual variables from their corresponding optimal solutions, under arbitrary quantization schemes. The results in this subsection indicate that the distance between the optimization variables and the optimal solution cannot be made arbitrarily close to zero at a given time instance $k$. The following corollary presents a non-asymptotic lower bound on the MSD of the PD variables.

Corollary 1. [10] Consider the PD algorithm under the quantization scheme Q. Then, the MSD of the PD variables from the optimal solution at iteration $k$ can be lower bounded as

$$
\begin{aligned}
\log \mathrm{E}\left[\left\|\varepsilon_{k}\right\|_{2}^{2}\right] & \geq \log \left(\frac{\mathrm{e}^{1-\frac{1}{M+N}}}{2 \pi \mathrm{e}}\right)+\frac{2}{N+M}\left(\sum_{i=1}^{M} \sum_{n=0}^{k-1} \log \left(1+\mu_{n} U_{i}^{\min }\right)\right. \\
& \left.+\mathrm{h}\left[y_{0}\right]-\sum_{t=0}^{k-1}\left(\left(\sum_{i=1}^{M} \log \left|\mathscr{A}_{i, t}^{x}\right|\right)+\sum_{j=1}^{N} \log \left|\mathscr{A}_{j, t}^{\lambda}\right|\right)\right),
\end{aligned}
$$

Corollary 1 provides a universal lower bound on the MSD of PD variables under quantized communications between agents and NNs. This result indicates that at a given time the PD variables cannot be arbitrarily close to the optimal solution (in the mean square sense), and imposes a lower bound on the MSD of PD variables from the optimal solution at a given time. According to Corollary 1, the MSD of PD variables from the optimal solution at iteration $k$ is bounded from below by the behavior of the second derivative of the objective functions of agents along the trajectories of primal variables up to time $k-1$, the total number of bits exchanged between agents and NNs up to time $k-1$, the differential entropy of distribution of initial PD variables, i.e., $\mathrm{h}\left[y_{0}\right]$, and the number of constraints and agents. The impact of objective functions of agents and the data rate between agents and NNs on the lower bound in (15) are similar to those in Theorem 1.

Note that the entropy power of $y_{0}$, i.e., $\frac{1}{2 \pi \mathrm{e}} \mathrm{e}^{\frac{2}{N+M}} \mathrm{~h}\left[y_{0}\right]$ is a measure of effective support volume of the random vector $y_{0}$. Thus, as $\mathrm{h}\left[y_{0}\right]$ becomes large, the size of the effective support set of $y_{0}$ increases, i.e., $y_{0}$ will be distributed on a larger region of $\mathbb{R}^{N+M}$. As a result, the MSD of the PD variables from the optimal solution increases since $y_{0}$ effectively takes value from a larger set, a behavior predicted by Corollary 1 .

The next corollary establishes a lower bound on the MSD of primal and dual variables:

Corollary 2. [10] Let $\mathrm{E}\left[\left\|\varepsilon_{k}^{x}\right\|_{2}^{2}\right]$ and $\mathrm{E}\left[\left\|\varepsilon_{k}^{\lambda}\right\|_{2}^{2}\right]$ be the MSD of the primal variables and dual variables, respectively, at iteration $k$ from the optimal solution. Then, 


$$
\begin{aligned}
& \log \mathrm{E}\left[\left\|\varepsilon_{k}^{x}\right\|_{2}^{2}\right] \geq \log \left(\frac{\mathrm{e}^{1-\frac{1}{M}}}{2 \pi \mathrm{e}}\right)+\frac{2}{M}\left(\sum_{i=1}^{M} \sum_{n=0}^{k-1} \log \left(1+\mu_{n} U_{i}^{\min }\right)+\mathrm{h}\left[x_{0}\right]-\sum_{t=0}^{k-1} \sum_{j=1}^{N} \log \left|\mathscr{A}_{j, t}^{\lambda}\right|\right), \\
& \log \mathrm{E}\left[\left\|\varepsilon_{k}^{\lambda}\right\|_{2}^{2}\right] \geq \log \left(\frac{\mathrm{e}^{1-\frac{1}{N}}}{2 \pi \mathrm{e}}\right)+\frac{2}{N}\left(\mathrm{~h}\left[\lambda_{0}\right]-\sum_{t=0}^{k-1} \sum_{i=1}^{M} \log \left|\mathscr{A}_{i, t}^{x}\right|\right),
\end{aligned}
$$

\subsection{An Optimum Achieving Quantization Scheme for NUM}

This section presents a zoom-in uniform optimum achieving (OA) quantization scheme for the PD algorithm, denoted as $\mathscr{Q}_{\text {a }}$. It is also proven that the PD algorithm under the quantization scheme $\mathscr{Q}_{\text {a }}$ converges to the optimal solution of the optimization problem (1). To this end, assume that the unquantized PD algorithm forms a contraction map with contraction constant $\alpha \in[0,1)$. Further assume that $\alpha$ is known by all agents and NNs. Under the quantization scheme $\mathscr{Q}_{\mathrm{a}}$, the quantization step at iteration $k$, i.e., $\delta_{k}$, is set to $\delta_{k}=\alpha^{k+1}$.

At time $k=0$, the agent $i$ generates $x_{0}^{i}$ according to a uniform distribution on the interval $(-L \alpha, L \alpha)$ where $L$ is a positive integer. Similarly, NN $j$ generates $\lambda_{0}^{j}$ using a uniform distribution on $(-L \alpha, L \alpha)$. Next, agents and NNs quantize the initial primal and dual variables, respectively, using a midpoint uniform quantizer on $(-L \alpha, L \alpha)$ with quantization step $\delta_{0}=\alpha$. Thus, the quantizer employed by agents and NNs at time $k=0$, is given by $Q_{\mathrm{a}, 0}(z)=\left\lfloor\frac{z}{\alpha}\right\rfloor \alpha+\frac{\alpha}{2}$ for $z \in(-L \alpha, L \alpha)$ where $\lfloor\cdot\rfloor$ is the floor function. Each agent (NN) only needs $\left\lceil\log _{2}(2 L)\right\rceil$ bits to communicate its initial primal (dual) variable where $\lceil\cdot\rceil$ is the ceiling function.

At time $k+1$, agent $i$ first encodes $x_{k+1}^{i}$ using the encoder $\hat{Q}\left(\frac{x_{k+1}^{i}-C_{k+1}^{x^{i}}}{\delta_{k+1}}\right)$ where $C_{k+1}^{x^{i}}=Q_{i, k}^{x}+$ $\left\lfloor\frac{x_{k+1}^{i}-x_{k}^{i}}{\delta_{k}}\right\rfloor \delta_{k}, Q_{i, k}^{x}$ is the quantized version of $x_{k}^{i}$, and $\hat{\mathrm{Q}}(\cdot)$ is given by

$$
\hat{\mathrm{Q}}(z)=\left\{\begin{array}{cc}
\left\lceil\frac{2}{\alpha}\right\rceil-1 & \left(\left\lceil\frac{2}{\alpha}\right\rceil-1\right) \leq z \leq\left\lceil\frac{2}{\alpha}\right\rceil \\
\lfloor z\rfloor-\left\lceil\frac{2}{\alpha}\right\rceil \leq z \leq\left(\left\lceil\frac{2}{\alpha}\right\rceil-1\right)
\end{array}\right.
$$

Let $I_{k+1}^{x^{i}}$ be the interval centered at $C_{k+1}^{x^{i}}$ with length $2\left\lceil\frac{2}{\alpha}\right\rceil \delta_{k+1}$. It can be shown that $x_{k+1}^{i}$ belongs to this interval which implies that the encoder mapping is always well defined (see the proof of Theorem 5 for more details).

Next, the agent $i$ transmits $\mathrm{Q}\left(\frac{x_{k+1}^{i}-C_{k+1}^{i}}{\delta_{k+1}}\right)$ to its neighboring NNs in the communication graph using $\left\lceil\log _{2}\left(2\left\lceil\frac{2}{\alpha}\right\rceil\right)\right\rceil$ bits. Agent $i$ also transmits $\left\lfloor\frac{x_{k+1}^{i}-x_{k}^{i}}{\delta_{k}}\right\rfloor$ to its neighboring NNs. This will allow the neighboring NNs of the agent $i$ to compute $C_{k+1}^{x^{i}}$, and update their decoders at time $k+1$. Note that $\left\lfloor\frac{x_{k+1}^{i}-x_{k}^{i}}{\delta_{k}}\right\rfloor$ is an integer which can be transmitted using finite number of bits. Finally, the neighboring NNs of agent $i$ construct the quantized version of $x_{k+1}^{i}$ using the decoder mapping $Q_{i, k+1}^{x}=C_{k+1}^{x^{i}}+\hat{\mathrm{Q}}\left(\frac{x_{k+1}^{i}-C_{k+1}^{x^{i}}}{\delta_{k+1}}\right) \delta_{k+1}+\frac{\delta_{k+1}}{2}$. 
At time $k+1, \mathrm{NN} j$ first encodes $\lambda_{k+1}^{j}$ using the encoder mapping $\hat{\mathrm{Q}}\left(\frac{\lambda_{k+1}^{j}-C_{k+1}^{\lambda^{j}}}{\delta_{k+1}}\right)$ where $C_{k+1}^{\lambda^{j}}=Q_{j, k}^{\lambda}+$ $\left\lfloor\frac{\lambda_{k+1}^{j}-\lambda_{k}^{j}}{\delta_{k}}\right\rfloor \delta_{k}, Q_{j, k}^{\lambda}$ is the quantized version of $\lambda_{k}^{j}$ and $\hat{\mathrm{Q}}(\cdot)$ is given by (16). Let $I_{k+1}^{\lambda^{j}}$ be the interval centered at $C_{k+1}^{\lambda^{j}}$ with the length $2\left\lceil\frac{2}{\alpha}\right\rceil \delta_{k+1}$. It can be shown that the $\lambda_{k+1}^{j}$ belongs to $I_{k+1}^{\lambda^{j}}$ which indicates that the encoder mapping is always well defined.

Next, NN $j$ transmits $\hat{\mathrm{Q}}\left(\frac{\lambda_{k+1}^{j}-C_{k+1}^{j}}{\delta_{k+1}}\right)$ and $\left\lfloor\frac{\lambda_{k+1}^{j}-\lambda_{k}^{j}}{\delta_{k}}\right\rfloor$ to its neighboring agents in the communication graph. Finally, the neighboring agents of the NN $j$ construct the quantized version of $\lambda_{k+1}^{j}$ using the decoder mapping $Q_{j, k+1}^{\lambda}=C_{k+1}^{\lambda^{j}}+\hat{\mathrm{Q}}\left(\frac{\lambda_{k+1}^{j}-C_{k+1}^{j}}{\delta_{k+1}}\right) \delta_{k+1}+\frac{\delta_{k+1}}{2}$.

The next theorem shows that the quantized PD algorithm under $\mathscr{Q}_{\mathrm{a}}$ converges to the optimal solution.

Theorem 5. [10] The PD algorithm under the quantization scheme $\mathscr{Q}_{\mathrm{a}}$ converges exponentially to the optimal solution of the optimization problem (1).

\section{Gradient-based Nash Seeking Algorithms Under Quantized Communications}

Game theory has been established to be of ubiquitous importance in engineering and used to analyze numerous problems, e.g., power control in wireless networks [11], wind energy harvesting, or sensor coverage [12]. In non-cooperative games, multiple agents aim to maximize individual utility functions by taking actions that are not necessarily coordinated with one another. The Nash equilibrium (NE) is one of the most important solution concepts in such games.

The problem of finding Nash equilibria is an active research area that has attracted much attention, e.g., see [13] and [14] and references therein. Gradient-based equilibrium-seeking (ES) algorithms are popular techniques for finding the $\mathrm{NE}$ of games with continuous action spaces and differentiable utility functions. In such algorithms, each agent modifies its current action according to the partial derivative of its utility function with respect to its action. The computation of this derivative implicitly requires communication between agents, since it typically depends on the actions of other agents.

This section investigates the effect of quantized communication on gradient-based, Nash-seeking algorithms. More specifically, the following questions are addressed: $(i)$ How does the communication data rate generally affect the convergence speeds achievable by ES algorithms? (ii) Given a uniform quantization scheme, on average how many time-steps are required for the ES algorithm to settle inside a ball around the NE? (iii) Given a uniform quantization scheme, what is the probability that agents' actions lie outside this ball at a given time? It is a point in the action space at which no agent can increase its own utility by unilaterally changing its action.

Subsection 3.1 introduces the non-cooperative game among agents and the distributed Nash seeking algorithm under quantized communications. The main results on the asymptotic and non-asymptotic behaviors of the Nash seeking algorithm are discussed in Subsection 3.2. 


\subsection{Game Model}

Consider a game with $M$ agents, indexed by $i \in \mathscr{M}:=\{1, \cdots, M\}$. Let $x^{i} \in \mathbb{R}$ be the action of the $i$-th agent, $x^{-i}:=\left[x^{1}, \cdots, x^{i-1}, x^{i+1}, x^{M}\right]^{\top} \in \mathbb{R}^{M-1}$, the vector of all agents actions except the $i$-th, and $U_{i}\left(x^{i}, x^{-i}\right) \in \mathbb{R}$ the utility of the $i$-th agent. Refer to this game as $\mathscr{G}=\left\langle\mathscr{M},\left\{x^{i}\right\}_{i},\left\{U_{i}(\cdot)\right\}_{i}\right\rangle$. Assume that each utility function $U_{i}\left(x^{i}, x^{-i}\right)$ is twice continuously differentiable and concave with respect to $x^{i}$.

Ideally, each agent in the game would like to make its own utility as large as possible. However, since the global maximizers of the utility functions will not generally coincide, a compromise is needed. This is provided by the Nash equilibrium $(N E)$, already. If all agents play their NE strategies, denoted by $x_{\mathrm{NE}}^{i}$, $i \in \mathscr{M}$, then no agent can increase its individual utility by unilaterally changing its action, i.e.

$$
x_{\mathrm{NE}}^{i}=\arg \max _{x^{i}} U_{i}\left(x^{i}, x_{\mathrm{NE}}^{-i}\right), \forall i \in \mathscr{M} .
$$

Throughout this section, it is assumed that the game admits a unique NE. This can be easily satisfied by imposing some additional mild conditions on the utility functions of agents, e.g., see [15]. Games arising in many engineering applications often admit a unique NE, which is associated with the desired operating point.

\subsubsection{Gradient-based Equilibrium Seeking with Quantized Communication}

Gradient-based, equilibrium seeking (ES) algorithms are among the most popular iterative techniques for finding the NE of a game with continuous action spaces and differentiable utility functions. In the absence of quantization, such algorithms take the general form

$$
x_{k+1}^{i}=x_{k}^{i}+\mu_{k} \frac{\partial}{\partial x^{i}} U_{i}\left(x_{k}^{i}, x_{k}^{-i}\right), k \in \mathbb{N}_{0}:=\{0,1,2 \ldots\}
$$

where $x_{k}^{i}$ is the action of the $i$-th agent at iteration $k, x_{k}^{-i}$ is the vector of all agent actions at iteration $k$ except the $i$-th, and $\mu_{k}>0$ is a time-varying step size.

In order to implement this update rule, each agent does not need to know other agents' utility functions, which may be kept private, but only their latest actions. However, agents in a distributed game are often located far from each other, e.g. power plants competing in a wholesale electricity market for maximizing their individual profits. The long distance between agents, combined with finite transmission power and bandwidth, limit the communication capacity between agents. Consequently, the agents in distributed games cannot transmit their actions with infinite resolution, but instead exchange quantized versions that are representable with finite numbers of bits.

Assuming that each $i$-th agent knows its own action $x_{k}^{i}$ perfectly, let $D_{i, k}\left(x_{k}^{i}\right) \in \mathscr{A}_{i, k}$ represent the quantized action broadcast by it to all other agents at iteration $k$. Here $\mathscr{A}_{i, k} \subset \mathbb{R}$ is a finite set and $\left|\mathscr{A}_{i, k}\right|$ is the number of quantization levels used by the $i$-th agent at iteration $k$. A large value of $\left|\mathscr{A}_{i, k}\right|$ implies that the $i$-th agent transmits its action with high precision, whereas a low value reflects poor communication capacity and low precision.

Let $D_{k}\left(x_{k}\right) \in \mathscr{A}_{k}$ represent the component-wise quantized version of the vector $x_{k}$, that is $D_{k}\left(x_{k}\right)=$ $\left[D_{i, k}\left(x_{k}^{i}\right)\right]_{i}^{\top}$. Note that $\log \left|\mathscr{A}_{k}\right|$ denotes the aggregate number of bits used by agents to represent their actions in the $k$-th iteration, where $\left|\mathscr{A}_{k}\right|=\prod_{i=1}^{M}\left|\mathscr{A}_{i, k}\right|$. With a slight abuse of notation, let $Q_{k}$ denote the quantized 
version of the vector $x_{k}^{-i}$. The ES algorithm with quantization then takes the form

$$
x_{k+1}^{i}=x_{k}^{i}+\mu_{k} \frac{\partial}{\partial x^{i}} U_{i}\left(x_{k}^{i}, Q_{k}\right), \quad \forall i \in \mathscr{M} .
$$

Let $\mathscr{D}=\left\{D_{k}\right\}_{k=0}^{\infty}$ be a quantization scheme. Given $\mathscr{D}$, the average aggregate data rate per unit time is defined as

$$
R_{\mathscr{D}}:=\limsup _{k \longrightarrow \infty} \frac{1}{k} \sum_{j=0}^{k-1} \log \left|\mathscr{A}_{j}\right| .
$$

The next subsection studies the asymptotic and transient performance of this algorithm.

\subsection{Equilibrium-seeking Algorithm Results and Discussion}

This section presents three performance measures for gradient-based equilibrium-seeking (ES) algorithms under quantization (18) : $(i)$ the asymptotic rate of exponential mean-square convergence to the Nash equilibrium (NE), (ii) the expected time for agent actions to settle inside a specified neighborhood of the NE, under uniform quantization, and (iii) the probability that agent actions at a given iteration $k$ lie outside this neighborhood, also under uniform quantization. The first criterion measures the long-term performance of the system, whereas the other two criteria characterize its transient performance. It is assumed that $x_{0}$, the vector of initial agent actions, is drawn randomly according to a probability distribution on $\mathbb{R}^{M}$. This assumption allows application of stochastic methods to analyze performance, under mild assumptions on the initial distribution.

\subsubsection{Lower Bound on the Asymptotic Mean Square Convergence Rate}

This subsection presents a universal lower bound on the asymptotic convergence rate of any quantized ES scheme of the form (18). In this asymptotic analysis, it is assumed that

- the joint probability density function (pdf) $p_{x_{0}}$ of initial actions has finite differential entropy, i.e. $\left|-\int p_{x_{0}}\left(x_{0}\right) \log \left(p_{x_{0}}\left(x_{0}\right)\right) d x_{0}\right|<\infty$.

- the second partial derivatives of the utility functions are bounded above and below as

$$
c_{i} \leq \frac{\partial^{2}}{\partial x^{i^{2}}} U_{i}\left(x^{i}, x^{-i}\right) \leq b_{i}<0, \quad \forall x \in \mathbb{R}^{M}
$$

- the step sizes $\mu_{k}>0$ converge to $\mu^{\star}>0$ as $k \rightarrow \infty$ and also satisfy $\sup _{k \in \mathbb{N}_{0}} \mu_{k}<\frac{1}{\max _{i}\left|c_{i}\right|}$, where $c_{i}$ is the lower bound (20) on the second derivative of the $i$-th agent's utility function with respect to its action.

Next, equilibrium achieving (EA) quantization schemes are defined.

Definition 4. A quantization scheme $\mathscr{D}$ is equilibrium-achieving if all quantized and unquantized actions converge to the NE with time for any initial condition in the support of $p_{x_{0}}$, i.e. 


$$
\begin{aligned}
& \lim _{k \longrightarrow \infty} x_{k}=x_{\mathrm{NE}}, \\
& \lim _{k \longrightarrow \infty} Q_{k}=x_{\mathrm{NE}} .
\end{aligned}
$$

The notion of distance decay exponent (DDE) for the ES algorithm under an EA quantization scheme is defined next.

Definition 5. For a given equilibrium-achieving quantization scheme $\mathscr{D}$, let $\varepsilon_{k}$ be the difference between agent actions and the $\mathrm{NE}$ at iteration $k$, i.e., $\varepsilon_{k}=x_{k}-x_{\mathrm{NE}}$. Then the distance decay exponent $(D D E)$ is defined as

$$
\liminf _{k \rightarrow \infty} \frac{1}{k} \log \mathrm{E}\left[\left\|\varepsilon_{k}\right\|_{2}^{2}\right] .
$$

The DDE gives the speed of exponential mean-square convergence of the agents' actions to NE under $\mathscr{D}$, where the expectation is taken with respect to the initial distribution of actions. A more negative exponent indicates faster convergence. The first main result of this section is stated now:

Theorem 6. [4] Let $\mathscr{D}$ be any equilibrium-achieving quantization scheme with average aggregate data rate $R_{\mathscr{D}}(19)$. Then, the error decay exponent is lower-bounded as

$$
\liminf _{k \rightarrow \infty} \frac{1}{k} \log \mathrm{E}\left[\left\|\varepsilon_{k}\right\|_{2}^{2}\right] \geq \frac{2}{M}\left(\sum_{i=1}^{M} \log \left(1+\left.\mu^{\star} \frac{\partial^{2} U_{i}}{\partial x^{i^{2}}}\right|_{x_{\mathrm{NE}}}\right)-R_{\mathscr{D}}\right) .
$$

Theorem 6 establishes a universal lower bound on the rate of exponential mean-square convergence that holds for any EA quantization scheme. This lower bound depends on the average aggregate date-rate $R_{\mathscr{D}}$, the second derivatives of the utility functions at the NE, and the number of agents. Recall that a more negative DDE corresponds to faster convergence.

Based on (22), the lower bound decreases (linearly) as $R_{\mathscr{D}}$ increases. This reflects the fact that each agent has more accurate information about the actions of the others and hence can make better decisions. Furthermore, the bound increases with the second derivatives of the utility functions. This is because a less negative second derivative indicates a flatter utility function, hence slower convergence to the NE.

Though the bound above may be conservative, unlike previous work it does not impose any particular structure on the quantization scheme, and delineates a universal trade-off between convergence rate, utility functions, data rate, and the number of agents.

\subsubsection{Transient Performance}

This subsection investigates the transient behavior of the equilibrium-seeking (ES) algorithm (18) under a uniform, time-invariant quantization scheme. The following assumptions are made on the game $\mathscr{G}$ and the ES algorithm (17):

- The NE of the game $\mathscr{G}$ belongs to the open, bounded and connected set $\mathscr{R} \subset \mathbb{R}^{M}$ which has non-zero Lebesgue measure.

- $x_{0}$, i.e, the initial action of agents, is randomly drawn from $\mathscr{R}$.

- $\frac{\partial}{\partial x^{i}} U_{i}\left(x^{i}, x^{-i}\right)$ is twice continuously differentiable for all $i$. 
- The ES algorithm under perfect communication, i.e., the update rule (17), is a pseudo-contraction mapping. That is

$$
\left\|x_{k}-x_{\mathrm{NE}}\right\|_{2} \leq \alpha\left\|x_{k-1}-x_{\mathrm{NE}}\right\|_{2}
$$

where $\alpha \in[0,1)$ and

$$
x_{k}^{i}=x_{k-1}^{i}+\mu_{k-1} \frac{\partial}{\partial x^{i}} U_{i}\left(x_{k-1}^{i}, x_{k-1}^{-i}\right)
$$

- The sequence $\left\{\mu_{k}\right\}_{k=0}^{\infty}$ is assumed to be bounded.

Let $d$ be the diameter of $\mathscr{R}$, that is, $d=\sup \left\{\|x-y\|_{2}: x, y \in \mathscr{R}\right\}$. Let $\mathrm{B}\left(x_{c}, d / 2\right)$ be the smallest ball containing $\mathscr{R}$ where $\mathrm{B}\left(x_{c}, d / 2\right)$ represents a closed ball in Euclidean norm centered at $x_{c}$ with radius $d / 2$. Let $\mathrm{Q}\left(x_{c}, 3 d / 2\right)$ be the cube centered at $x_{c}$ with side length $3 d$. In this subsection, assume that the agents employ a uniform, time-invariant quantization scheme denoted by $\mathscr{D}_{\mathrm{u}}$. Under $\mathscr{D}_{\mathrm{u}}$, the intersection of $\mathrm{Q}\left(x_{c}, 3 d / 2\right)$ and action space of each agents is uniformly quantized with the quantization step $\delta$. The ES update rule under the uniform quantization scheme $\mathscr{D}_{\mathrm{u}}$ is given by

$$
x_{k+1}^{i}=x_{k}^{i}+\mu_{k} \frac{\partial}{\partial x^{i}} U_{i}\left(x_{k}^{i}, \mathscr{D}_{\mathrm{u}}\left(x_{k}^{-i}\right)\right), \forall i .
$$

Since the quantization scheme $\mathscr{D}_{\mathrm{u}}$ is only defined on $\mathrm{Q}\left(x_{c}, 3 d / 2\right)$, the actions of agents have to stay in $\mathrm{Q}\left(x_{c}, 3 d / 2\right)$. Note that if $x_{0}$, the initial action of agents, belongs to $\mathscr{R}$ and the quantization step $\delta$ is sufficiently small, then, the actions of agents will always stay in $\mathrm{Q}\left(x_{c}, 3 d / 2\right)$. A sufficient condition for $\delta$ is given by

$$
\begin{aligned}
\sup _{k} \mu_{k} & \left(\delta \sqrt{\sum_{i} \Phi_{i}^{2}}+\frac{1}{2} \delta^{2}\left(M \sqrt{\sum_{i} \Psi_{i}^{2}}+\sqrt{\sum_{i} \eta_{i}^{2}}\right)\right) \\
\leq & (1-\alpha) d,
\end{aligned}
$$

where $\Phi_{i}, \Psi_{i}$ and $\eta_{i}$ are given by

$$
\begin{aligned}
& \Phi_{i}=\sup _{x \in \mathrm{Q}\left(x_{c}, 3 d / 2\right)} \sum_{j \neq i}\left|\frac{\partial^{2}}{\partial^{2} x^{j} x^{i}} U_{i}\left(x^{i}, x^{-i}\right)\right|, \\
& \Psi_{i}=\sup _{x \in \mathrm{Q}\left(x_{c}, 3 d / 2\right)}\left\|\nabla^{2} \frac{\partial}{\partial x^{i}} U_{i}\left(x^{i}, x^{-i}\right)\right\|_{2}, \\
& \eta_{i}=\sup _{x \in \mathrm{Q}\left(x_{c}, 3 d / 2\right)}\left|\frac{\partial^{3}}{\partial x^{i^{3}}} U_{i}\left(x^{i}, x^{-i}\right)\right| .
\end{aligned}
$$

respectively, where $\nabla^{2}(\cdot)$ is the Hessian operator. The left hand side of (24) is an upper bound on the distortion induced by the quantization scheme $\mathscr{D}_{\mathrm{u}}$. Thus, (24) essentially implies that the quantization scheme $\mathscr{D}_{\mathrm{u}}$ is well defined if the distortion caused by the quantization scheme at each time step is small enough.

The term $\Phi_{i}$ represents the sensitivity of update rule of the $i$-th agent to the actions of other agents. When agents are less sensitive to each other's actions, according to (24), a relatively large quantization step $\delta$ can be chosen without introducing a large amount of distortion in the evolution of the ES algorithm. However, when 
agents are highly sensitive to each other's actions, a high resolution quantization scheme should be employed to avoid a large amount of distortion. Moreover, according to (24), small values of the step size result in small distortion values. Since each agent modifies its action by adding the term $\mu_{k} \frac{\partial}{\partial x^{i}} U_{i}\left(x_{k}^{i}, \mathscr{D}_{\mathrm{u}}\left(x_{k}^{-i}\right)\right)$ to its previous action, a small value of step size results in a small value of distortion at the cost of a slow convergence speed.

Let $\mathrm{E}[\mathscr{N}]$ denote the expected time required for $x_{k}$ to settle inside $\mathrm{B}\left(x_{\mathrm{NE}}, r\right)$. A small value of $\mathrm{E}[\mathscr{N}]$ indicates that the ES algorithm, on average, quickly approaches the NE whereas a large value of the $\mathrm{E}[\mathscr{N}]$ indicates a relatively slow convergence. Due to the quantization distortion, the radius of $\mathrm{B}\left(x_{\mathrm{NE}}, r\right)$ cannot be arbitrarily small. If $r$ is less than the total quantization distortion, one cannot guarantee that agents' actions will eventually settle inside $\mathrm{B}\left(x_{\mathrm{NE}}, r\right)$ as $k$ becomes large. Here, it is assumed that $r>\theta$ where $\theta$ is given by

$$
\theta=\frac{\sup _{k} \mu_{k}}{1-\alpha}\left(\delta \sqrt{\sum_{i} \Phi_{i}^{2}}+\frac{1}{2} \delta^{2}\left(M \sqrt{\sum_{i} \Psi_{i}^{2}}+\sqrt{\sum_{i} \eta_{i}^{2}}\right)\right) .
$$

Note that, $\theta$ represents an upper bound on the aggregate distortion caused by the quantization scheme $\mathscr{D}_{\mathrm{u}}$ over time (see [4] for more details). The next theorem provides an upper bound on $\mathrm{E}[\mathscr{N}]$.

Theorem 7. [4] Consider the uniform quantization scheme $\mathscr{D}_{\mathrm{u}}$ with the quantization step $\delta$ satisfying (24). Let $\mathrm{E}[\mathscr{N}]$ denote the expected time required for the ES algorithm under $\mathscr{D}_{\mathrm{u}}$ to settle in $\mathrm{B}\left(x_{\mathrm{NE}}, r\right)$ with $r>\theta$. Then, $\mathrm{E}[\mathscr{N}]$ is upper bounded as

$$
\mathrm{E}[\mathscr{N}] \leq \frac{1}{\log \left(\frac{1}{\alpha}\right)}\left(\mathrm{E}\left[\log \left(\frac{\left\|x_{0}-x_{\mathrm{NE}}\right\|_{2}}{r-\theta}\right) \mathrm{I}_{\left.\left\{\frac{\left\|x_{0}-x_{\mathrm{NE}}\right\|_{2}}{r-\theta}>1\right\}\right]}\right\}\right.
$$

Theorem 7 provides an upper bound on the expected time required for the actions of agents to settle inside a ball of radius $r$ centered at the NE. This upper bound is controlled by $\alpha, \theta, r$ and the distribution of the initial actions of agents. According to this theorem, the effect of $\alpha$ on the expected time is manifested through the multiplicative factor $\frac{1}{\log \left(\frac{1}{\alpha}\right)}$ with $\alpha \in[0,1)$. As $\alpha$ becomes closer to zero, the distance between the actions of agents and the NE decays faster due to the pseudo-contraction property of the non-quantized update rule. Thus, the average time required to settle inside $\mathrm{B}\left(x_{\mathrm{NE}}, r\right)$ becomes smaller as $\alpha$ decreases.

The function $\mathrm{E}[\mathscr{N}]$ is non-increasing in $r$. That is, as $r$ becomes small, it takes more time for the ES algorithm (23) to settle in $\mathrm{B}\left(x_{\mathrm{NE}}, r\right)$. This observation is also consistent with our result in Theorem 7, i.e., the upper bound on the $\mathrm{E}[\mathscr{N}]$ increases as $r$ becomes small. Finally, Theorem 7 suggests that the expected time required to settle inside $\mathrm{B}\left(x_{\mathrm{NE}}, r\right)$ is influenced by the distribution of initial actions of agents, $p_{x_{0}}(x)$. Observe that, when $p_{x_{0}}(x)$ is highly concentrated around the Nash equilibrium, the ES algorithm (23) requires less time to settle inside $\mathrm{B}\left(x_{\mathrm{NE}}, r\right)$ compared to the case that $p_{x_{0}}(x)$ has a low degree of concentration around the NE.

The upper bound in Theorem 7 depends on the distance between the initial action of agents and the NE, i.e., $\left\|x_{0}-x_{\mathrm{NE}}\right\|_{2}$. Since both $x_{0}$ and $x_{\mathrm{NE}}$ belong to $\mathscr{R}$, one can use the fact that $\left\|x_{0}-x_{\mathrm{NE}}\right\|_{2} \leq d$ to obtain an upper bound on the $\mathrm{E}[\mathscr{N}]$ which is independent of $x_{0}$ and $x_{\mathrm{NE}}$. This result is stated in the next corollary.

Corollary 3. [4] The expected time required for the ES algorithm under $\mathscr{D}$ to settle in $\mathrm{B}\left(x_{\mathrm{NE}}, r\right)$ can be upper bounded as

$$
\mathrm{E}[\mathscr{N}] \leq \frac{1}{\log \left(\frac{1}{\alpha}\right)} \log \left(\frac{d}{r-\theta}\right)
$$


Another performance measure for the transient behavior of the ES algorithm (23) under the uniform quantization scheme $\mathscr{D}_{\mathrm{u}}$ is investigated next. In this case, the probability that $x_{k}$ lies outside a ball of radius $r>\theta$ around the NE, i.e.,

$$
\operatorname{Pr}\left\{\left\|x_{k}-x_{\mathrm{NE}}\right\|_{2}>r\right\} .
$$

Note that, $\operatorname{Pr}\left\{\left\|x_{k}-x_{\mathrm{NE}}\right\|_{2}>r\right\}$ is a function of $k$, and decays to zero as $k$ tends to infinity. For a given $k$, a small value of $\operatorname{Pr}\left\{\left\|x_{k}-x_{\mathrm{NE}}\right\|_{2}>r\right\}$ indicates that $x_{k}$ approaches the NE at a higher speed compared to a large value of $\operatorname{Pr}\left\{\left\|x_{k}-x_{\mathrm{NE}}\right\|_{2}>r\right\}$. The next theorem provides an upper bound on the probability that $x_{k}$ lies outside $\mathrm{B}\left(x_{\mathrm{NE}}, r\right)$.

Theorem 8. [4] Consider the uniform quantization scheme $\mathscr{D}_{\mathrm{u}}$ with the quantization step $\delta$ satisfying (24). Then, the probability that $x_{k}$ lies outside $\mathrm{B}\left(x_{\mathrm{NE}}, r\right)$ with $r>\theta$ is upper bounded as

$$
\operatorname{Pr}\left\{\left\|x_{k}-x_{\mathrm{NE}}\right\|_{2}>r\right\} \leq \min \left(1, \mathrm{e}^{-\frac{r-\theta}{\alpha^{k}}} \mathrm{E}\left[\mathrm{e}^{\left\|x_{0}-x_{\mathrm{NE}}\right\|_{2}}\right]\right) .
$$

Theorem 8 provides an upper bound on the probability that $x_{k}$ lies outside of the ball radius $r$ around the $\mathrm{NE}$ at a given time. According to Theorem 8 , this probability decays to zero at least double exponentially with $k$. Also, the decay rate of this probability depends on the contraction constant $\alpha$. As $\alpha$ becomes small, the distance between agents' actions and the NE decays faster. Hence, the probability that $x_{k}$ lies outside $\mathrm{B}\left(x_{\mathrm{NE}}, r\right)$ decays faster to zero, and $x_{k}$ with high probability lies inside $\mathrm{B}\left(x_{\mathrm{NE}}, r\right)$. The term $\mathrm{E}\left[\mathrm{e}\left\|x_{0}-x_{\mathrm{NE}}\right\|_{2}\right]$ in (25) indicates the effect of the distribution of $x_{0}$ on $\operatorname{Pr}\left\{\left\|x_{k}-x_{\mathrm{NE}}\right\|_{2}>r\right\}$. That is, when the distribution of $x_{0}$ is more concentrated around the NE, we expect $x_{k}$ to approach the NE at a faster speed. Note that for a given $k, \operatorname{Pr}\left\{\left\|x_{k}-x_{\mathrm{NE}}\right\|_{2}>r\right\}$ is a non-increasing function of $r$. That is, $x_{k}$ lies outside $\mathrm{B}\left(x_{\mathrm{NE}}, r\right)$ with high probability as $r$ becomes small. This behavior is consistent with the upper bound in Theorem 8 .

One can use the fact that $\left\|x_{0}-x_{\mathrm{NE}}\right\|_{2} \leq d$ to obtain an upper bound on $\operatorname{Pr}\left\{\left\|x_{k}-x_{\mathrm{NE}}\right\|_{2}>r\right\}$ which is independent of $x_{0}$ and $x_{\mathrm{NE}}$. This result is stated in the next corollary.

Corollary 4. The probability that $x_{k}$ lies outside $\mathrm{B}\left(x_{\mathrm{NE}}, r\right)$ can be upper bounded as

$$
\operatorname{Pr}\left\{\left\|x_{k}-x_{\mathrm{NE}}\right\|_{2}>r\right\} \leq \min \left(1, \mathrm{e}^{d-\frac{r-\theta}{\alpha^{k}}}\right) .
$$

Finally, the upper bound in Corollary 4 can be used to obtain an upper bound on the number of time-steps required for $x_{k}$ to lie in $\mathrm{B}\left(x_{\mathrm{NE}}, r\right)$ with a given certainty level. Let $N_{p}$ be the number of time-steps required for $x_{k}$ to lie in B $\left(x_{\mathrm{NE}}, r\right)$ with the probability at least equal to $p$. Then, using (26), $N_{p}$ can be upper bounded by the smallest positive integer satisfying

$$
d-(r-\theta) \leq \alpha^{k} \log (1-p) .
$$

Theorem 8 and Corollary 4 can be used to obtain bounds on the required number of quantization levels to guarantee that the agents' actions at iteration $k$ lie inside a ball of radius $r$ around the NE with a given probability. In addition, Theorem 7 and Corollary 3 give rise to bounds on the required number of quantization levels to guarantee an average settling time. Finally, Theorem 6 provides guidelines on the required average aggregate data rate for achieving a desired speed of exponential convergence to the NE. 


\subsubsection{An Adaptive EA Quantization Scheme}

Based on the previous analysis of the uniform quantization scheme $\mathscr{D}_{\mathrm{u}}$, this subsection presents an adaptive EA quantization scheme under which the ES algorithm converges to the NE. This quantization scheme is denoted as $\mathscr{D}_{\mathrm{a}}$. Later, in the numerical result section, the error decay exponent of $\mathscr{D}_{\mathrm{a}}$ is studied. Recall that, the NE belongs to the region $\mathscr{R}$ with the diameter $d$. The basic idea behind the adaptive quantization scheme $\mathscr{D}_{\mathrm{a}}$ is to reduce the size of the known region around the NE in each time-step.

Let $\mathscr{R}_{k}$ denote the region which the NE belongs to at iteration $k$ under the quantization scheme $\mathscr{D}_{\text {a }}$. The quantization scheme $\mathscr{D}_{\mathrm{a}}$ is designed such that the diameter of $\mathscr{R}_{k}$ converges to zero as $k$ tends to infinity. Under the quantization scheme $\mathscr{D}_{\mathrm{a}}$, initially, the intersection of action space of each agent with $\mathrm{Q}\left(x_{c}, 3 d / 2\right)$ is quantized with the quantization step $\delta_{0}$ which satisfies the following inequality

$$
\sup _{k} \mu_{k}\left(\delta_{0} \sqrt{\sum_{i} \Phi_{i}^{2}}+\frac{1}{2} \delta_{0}^{2}\left(M \sqrt{\sum_{i} \Psi_{i}^{2}}+\sqrt{\sum_{i} \eta_{i}^{2}}\right)\right) \leq \hat{\alpha} d,
$$

where $\hat{\alpha}$ is a constant arbitrarily selected from the interval $(0,1-\alpha)$. It is straightforward to show that the distance between $x_{1}$ and the NE under $\mathscr{D}_{\mathrm{a}}$ can be upper bounded as

$$
\begin{aligned}
\left\|x_{1}-x_{\mathrm{NE}}\right\|_{2} & \leq \alpha\left\|x_{0}-x_{\mathrm{NE}}\right\|_{2}+\sup _{k} \mu_{k}\left(\delta_{0} \sqrt{\sum_{i} \Phi_{i}^{2}}+\frac{1}{2} \delta_{0}^{2}\left(M \sqrt{\sum_{i} \Psi_{i}^{2}}+\sqrt{\sum_{i} \eta_{i}^{2}}\right)\right) \\
& \leq(\alpha+\hat{\alpha}) d,
\end{aligned}
$$

which implies that the NE belongs to the ball of radius $(\alpha+\hat{\alpha}) d$ around $x_{1}$. In the second time-step, $\mathrm{Q}\left(x_{1},(\alpha+\hat{\alpha}) d\right) \cap \mathrm{Q}\left(x_{c}, 3 d / 2\right)$ is considered as $\mathscr{R}_{1}$ and the intersection of each agent's action space with the $\mathscr{R}_{1}$ is quantized. Similarly, at iteration $k, k \geq 1$, we have $\mathscr{R}_{k}=\mathrm{Q}\left(x_{k}, d_{k}\right) \cap \mathrm{Q}\left(x_{c}, 3 d / 2\right)$ where $d_{i}=(\alpha+\hat{\alpha}) d_{i-1}$ with $d_{0}=d$. Then, the intersection of action space of each agent with $\mathscr{R}_{k}$ is quantized. Also, the quantization step at iteration $k, \delta_{k}$, is chosen such that the following inequality is satisfied

$$
\sup _{k} \mu_{k}\left(\delta_{k} \sqrt{\sum_{i} \Phi_{i}^{2}}+\frac{1}{2} \delta_{k}^{2}\left(M \sqrt{\sum_{i} \Psi_{i}^{2}}+\sqrt{\sum_{i} \eta_{i}^{2}}\right)\right) \leq \hat{\alpha} d_{k},
$$

Since $d_{k}$ converges to zero as $k$ tends to infinity, the actions of agents and their quantized versions, under the quantization scheme $\mathscr{D}_{\mathrm{a}}$, converge to the NE as $k$ tends to infinity which implies that $\mathscr{D}_{\mathrm{a}}$ is an EA quantization scheme. Algorithm 1 shows the different steps of the adaptive quantization scheme $\mathscr{D}_{\mathrm{a}}$.

\subsection{Numerical Results}

This section presents a set of numerical results for a non-cooperative game with 5 agents seeking to maximize their utility functions. The utility function of $i$-th agent is given by

$$
U_{i}\left(x^{i}, x^{-i}\right)=\frac{t_{i i}}{2}\left(x^{i}\right)^{2}+x^{i}\left(\sum_{j \neq i} t_{i j} x^{j}-l_{i}\right),
$$




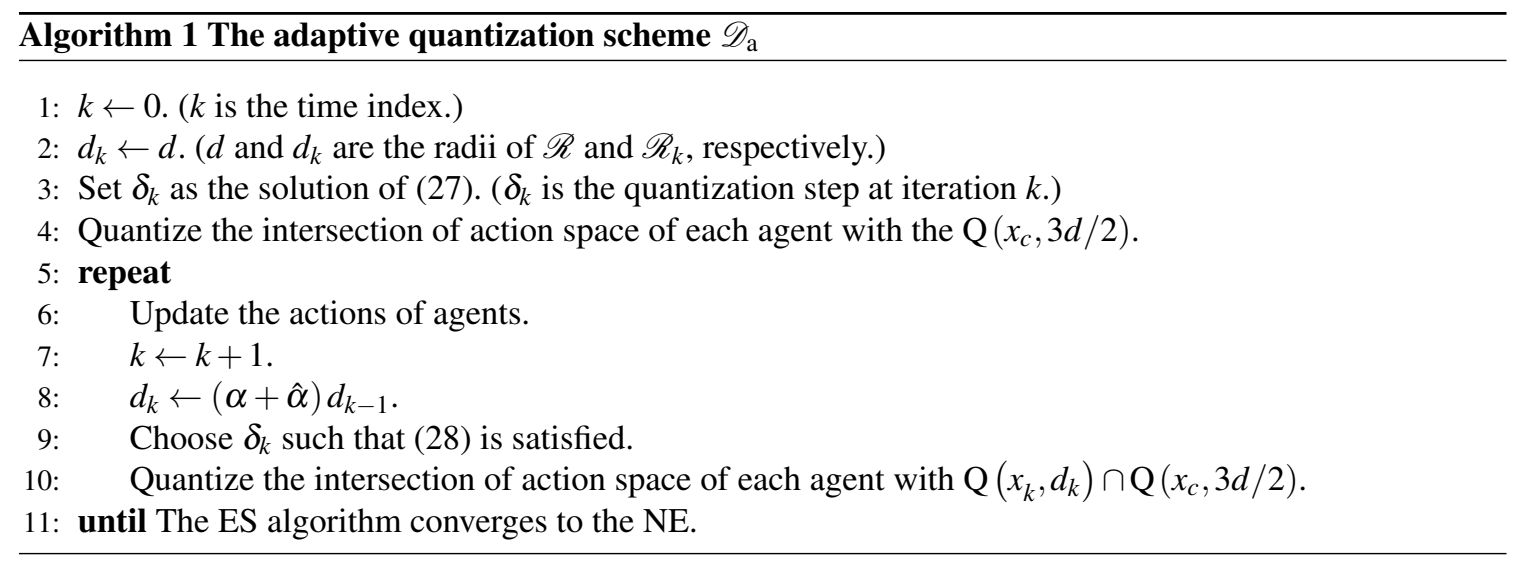

where $t_{i i}<0$ for all $i$ and $t_{i j}, l_{i} \in \mathbb{R}$. Utility functions of the form (29) arise in many engineering applications such as analyzing the bidding behavior of a group of generators competing for maximizing their profits in an electricity market, e.g., see [16]. Let $T$ be an $M$-by- $M$ matrix with the $(i, j)$-th entry equal to $t_{i j}$. Assume that $T$ is negative definite. Since $T$ is invertible, it can be easily verified, using the Karush-Kuhn-Tucker conditions, that the quadratic game with the utility functions (29) admits a unique Nash equilibrium. For this quadratic game, the ES algorithm under perfect communication condition can be written as

$$
x_{k+1}=(I+\mu T) x_{k}-\mu l,
$$

where $l=\left[l_{1}, \cdots, l_{M}\right]^{\top}$. The step size $\mu$ is selected such that the spectral radius of $I+\mu T$ is strictly less than one. In the numerical results, it is assumed that the NE belongs to a hypercube $\mathscr{R}$, whose side length is equal to $\frac{10}{\sqrt{5}}$. Additionally, the vector of initial actions of agents, $x_{0}$, is assumed to be uniformly distributed on $\mathscr{R}$.

Fig. 2 illustrates the expected time required for $x_{k}$ to settle inside $\mathrm{B}\left(x_{\mathrm{NE}}, r\right)$ as a function of $r$ for different quantization schemes and different values of average aggregate data rates $R_{\mathscr{D}}$. In this figure, $\theta, d$ and $\alpha$ are set to $10^{-2}, 10$ and 0.46 , respectively. As $r$ becomes large, the ES algorithm under both $\mathscr{D}_{\mathrm{u}}$ and $\mathscr{D}_{\mathrm{a}}$ requires less time to settle inside $\mathrm{B}\left(x_{\mathrm{NE}}, r\right)$, and as a result, the expected time, under both $\mathscr{D}_{\mathrm{u}}$ and $\mathscr{D}_{\mathrm{a}}$, decreases as $r$ becomes large. As shown in Fig. 2, the expected time under the fixed quantization scheme $\mathscr{D}_{\mathrm{u}}$ is limited by the upper bound provided by Theorem 7. According Fig. 2, the ES algorithm under the EA quantization scheme $\mathscr{D}_{\mathrm{a}}$, on average, requires less time to settle inside $\mathrm{B}\left(x_{\mathrm{NE}}, r\right)$ compared to the fixed quantization scheme $\mathscr{D}_{\mathrm{u}}$. The fast convergence of the ES algorithm under $\mathscr{D}_{\mathrm{a}}$ is due to the flexible structure of the EA quantization scheme $\mathscr{D}_{\mathrm{a}}$.

In Fig. 2, the ES algorithm under $\mathscr{D}_{\mathrm{u}}$ with coarse quantization scheme $R_{\mathscr{D}}=25$ cannot settle inside the ball $\mathrm{B}\left(x_{\mathrm{NE}}, r\right)$ when $r$ is small which is due to the large amount of distortion caused by the coarse quantization scheme. However, as $r$ becomes large, the ES algorithm under the coarse quantization scheme settles inside the ball $\mathrm{B}\left(x_{\mathrm{NE}}, r\right)$ faster than the fine quantization scheme $R_{\mathscr{D}}=60$. Note that any quantization scheme introduces an extra displacement to the agents' action at each time-step. A coarse quantization scheme causes a bigger displacement, compared to a fine quantization scheme, which results in a lower expected time to settle inside $\mathrm{B}\left(x_{\mathrm{NE}}, r\right)$ (when $r$ is large enough). 


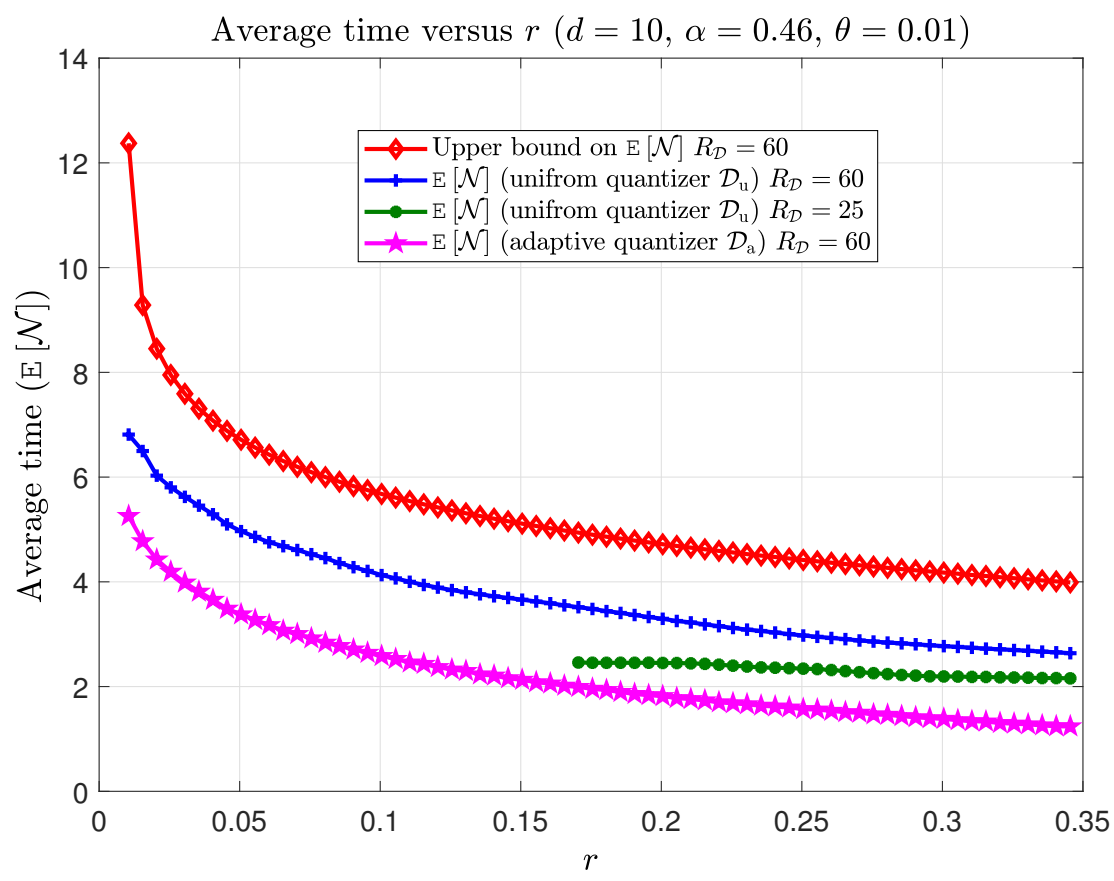

Fig. 2 The average time required for the ES algorithm to settle in a ball of radius $r$ around the NE for the fixed and adaptive quantizers as a function of $r$.

Fig. 3 demonstrates the behavior of log-mean-square-error-norm divided by $k$, i.e., $\frac{1}{k} \log \mathrm{E}\left[\left\|\varepsilon_{k}\right\|_{2}^{2}\right]$, under the adaptive quantizer $\mathscr{D}_{\mathrm{a}}$ as a function of the number of time-steps. As Fig. 3 shows $\frac{1}{k} \log \mathrm{E}\left[\left\|\varepsilon_{k}\right\|_{2}^{2}\right]$ stays above -4.2318 , the predicted lower bound by the Theorem 6 , as $k$ becomes large.

\section{Conclusion}

This chapter presented a set of results on the convergence behavior of a quantized primal-dual (PD) algorithm as well as a gradient-based Nash seeking algorithm under quantized inter-agent communications. First, using the information-theoretic notion of entropy power, universal bounds are derived on the fastest speed of exponential mean square convergence of PD, primal and dual variables to the optimal solution under optimum achieving quantization schemes. These results highlight the universal trade-offs between the speed of convergence of the quantized PD algorithm, data rate under the quantization, objective functions of agents, the number of agents, and the number of constraints. Next, universal lower bounds are established on the mean square distance of PD, primal and dual variables from the optimal solution of the NUM problem for any finite time index.

Subsequently, the impact of quantized inter-agent communications on the convergence behavior of the gradient-based Nash equilibrium seeking (ES) algorithm in non-cooperative games is studied. The 


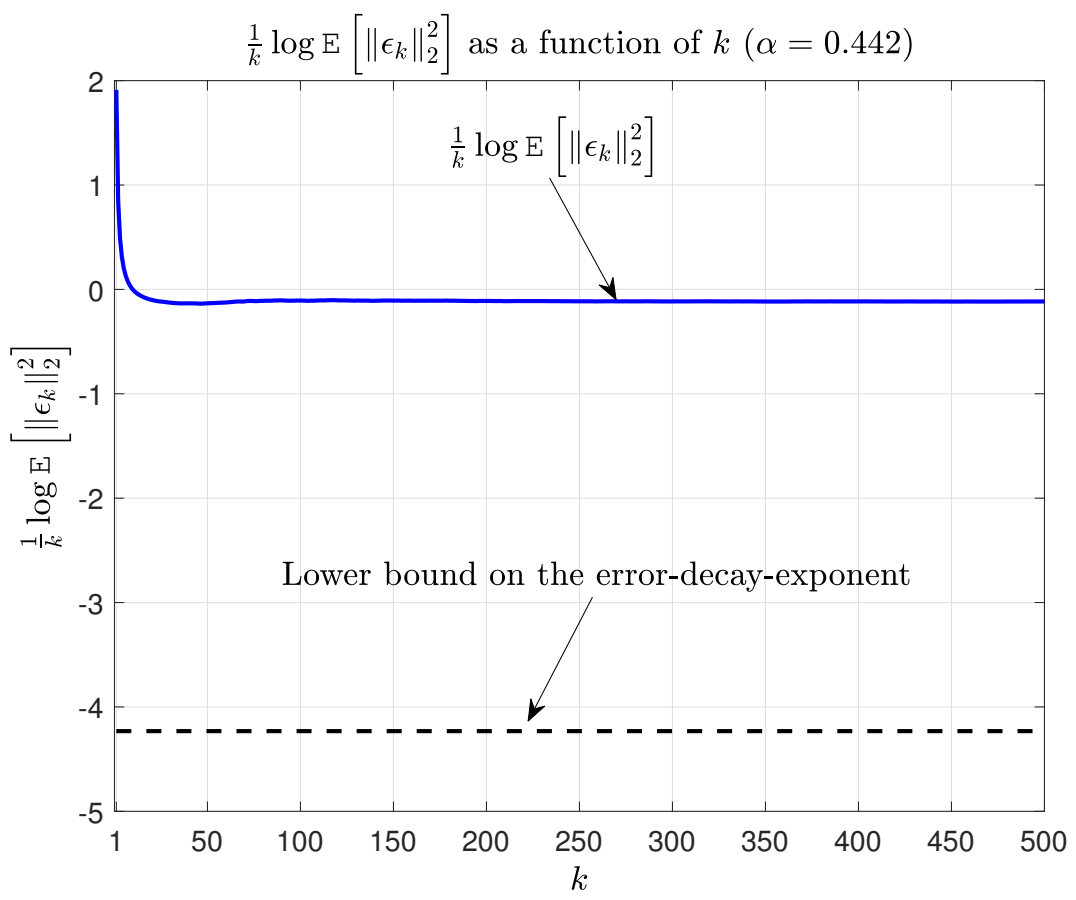

Fig. $3 \log$-mean-square-error-norm divided by $k$ under the adaptive quantization scheme $\mathscr{D}_{\mathrm{a}}$ as a function of $k$.

information-theoretic notion of entropy power helped establishing a universal lower bound on the rate of exponential mean square convergence of such algorithms, assuming equilibrium-achieving quantizers. This lower bound signifies the impact of inter-agent communication data rates on the convergence speed of the ES algorithm to the Nash equilibrium (NE). Next, the transient behavior of the ES algorithm under quantized message passing among agents was examined. To this end, an upper bound is derived on the expected time required for the ES algorithm to settle inside a ball centered at the NE under a uniform quantization scheme. Moreover, an upper bound is obtained on the probability that agents' actions at a given time lie outside a ball around the Nash equilibrium. It is worth noting that these last two results only concern the behavior of the ES algorithm until it reaches a neighborhood of the Nash equilibrium, and do not make any assumption on the convergence of the ES algorithm.

Acknowledgements The authors would like to thank Prof. Girish Nair from The University of Melbourne for his contributions and fruitful discussions. This work was supported by the Australian Research Councils Discovery Projects funding scheme (DP140100819). 


\section{Appendix}

\section{Proof of Theorem 1}

This appendix presents the main steps of the proof of Theorem 1. To this end, first, the notion of conditional differential entropy power of a random vector is defined. Then, the notion of entropy power facilitates establishing a universal lower bound on the DDE of the PD variables. The differential entropy power of the random vector $z \in \mathbb{R}^{N+M}$ conditioned on the event $A=a$, denoted by $\mathrm{N}[z \mid A=a]$, is defined as

$$
\mathrm{N}[z \mid A=a]=\frac{1}{2 \pi \mathrm{e}} \mathrm{e}^{\frac{2}{M+N} \mathrm{~h}[z \mid A=a]},
$$

where $\mathrm{h}[z \mid A=a]$ is the conditional differential entropy of $z$ given $A=a$ defined as

$$
\mathrm{h}[z \mid A=a]=-\int \log (p(z \mid A=a)) p(z \mid A=a) d z,
$$

where $p(z \mid A=a)$ is the conditional distribution of $z$ given $A=a$. Using the entropy maximizing property of Gaussian distributions, the conditional entropy power of $z$ given $A=a$ can be upper bounded [1] as

$$
\mathrm{N}[z \mid A=a] \leq \mathrm{e}^{1 /(M+N)-1} \mathrm{E}\left[\|z\|_{2}^{2} \mid A=a\right],
$$

where $\mathrm{E}[z \mid A=a]$ is conditional expectation of $z$ given $A=a$. Let $\mathrm{E}_{A}[\mathrm{~N}[z \mid A=a]]$ denote the average conditional entropy power of $z$ given $A=a$. Using (30), $\mathrm{E}_{A}[\mathrm{~N}[z \mid A=a]]$ can be upper bounded as

$$
\mathrm{E}_{A}[\mathrm{~N}[z \mid A]] \leq \mathrm{e}^{1 /(M+N)-1} \mathrm{E}\left[\|z\|_{2}^{2}\right] .
$$

Next, the inequality (31) is used to establish the universal lower bound on the DDE of the PD variables under OA quantization schemes. To this end, let $\mathscr{D}_{k-1}=\left\{\hat{Q}_{n}=\hat{q}_{n}\right\}_{n=0}^{k-1}$ where $\hat{Q}_{n}=\left[\hat{Q}_{1, n}^{x}, \cdots, \hat{Q}_{M, n}^{x}, \hat{Q}_{1, n}^{\lambda}, \cdots, \hat{Q}_{N, n}^{\lambda}\right]$ and $\hat{q}_{n}$ is a possible realization of $\hat{Q}_{n}$. Using (31), $\mathrm{E}\left[\left\|\varepsilon_{k}\right\|_{2}^{2}\right]$ can be lower bounded as $\frac{\mathrm{e}^{1-\frac{1}{M+N}}}{2 \pi \mathrm{e}} \mathrm{e}^{\frac{2}{M+N}} \mathrm{E}\left[\mathrm{h}\left[\varepsilon_{k} \mid \mathscr{V}_{k-1}\right]\right]$

$$
\begin{aligned}
\mathrm{E}\left[\left\|\varepsilon_{k}\right\|_{2}^{2}\right] & \geq \mathrm{e}^{1-\frac{1}{M+N}} \mathrm{E}\left[\mathrm{N}\left[\varepsilon_{k} \mid \mathscr{D}_{k-1}\right]\right] \\
& \stackrel{(*)}{\geq} \frac{\mathrm{e}^{1-\frac{1}{M+N}}}{2 \pi \mathrm{e}} \mathrm{e}^{\frac{2}{M+N}} \mathrm{E}\left[\mathrm{h}\left[\varepsilon_{k} \mid \mathscr{D}_{k-1}\right]\right]
\end{aligned}
$$

where $(*)$ is obtained using the Jensen inequality. The term $\mathrm{h}\left[\varepsilon_{k} \mid \mathscr{D}_{k-1}\right]$ on the right hand side of (32) can be expanded as

$$
\begin{aligned}
\mathrm{h}\left[\varepsilon_{k} \mid \mathscr{D}_{k-1}\right] & =\mathrm{h}\left[y_{k}-y^{\star} \mid \mathscr{D}_{k-1}\right] \\
& \stackrel{(*)}{=} \mathrm{h}\left[y_{k} \mid \mathscr{D}_{k-1}\right],
\end{aligned}
$$

where $(*)$ follows from the translation invariance property of differential entropy as $y^{\star}$ is a constant vector (see [17] Theorem 8.6.3 page 253). 
The next lemma establishes a useful expression between $\mathrm{h}\left[y_{n} \mid \mathscr{D}_{k-1}\right]$ and $\mathrm{h}\left[y_{n-1} \mid \mathscr{D}_{k-1}\right]$ for $n \leq k$, which is used to further expand $\mathrm{h}\left[y_{k} \mid \mathscr{D}_{k-1}\right]$.

Lemma 1. For $n \leq k, \mathrm{~h}\left[y_{n} \mid \mathscr{D}_{k-1}\right]$ can be expanded as

$$
\mathrm{h}\left[y_{n} \mid \mathscr{D}_{k-1}\right]=\mathrm{h}\left[y_{n-1} \mid \mathscr{D}_{k-1}\right]+\mathrm{E}\left[\sum_{j=1}^{M} \log \left(1+\mu_{n-1} \frac{d^{2}}{d x^{j}} U_{j}\left(x_{n-1}^{j}\right)\right) \mid \mathscr{D}_{k-1}\right]
$$

Proof. Let $\tilde{x}_{n}^{i}=x_{n}^{i}+\mu_{n}\left(\frac{d}{d x^{i}} U_{i}\left(x_{n}^{i}\right)\right)$ and $\tilde{x}_{n}=\left[\tilde{x}_{1}^{i}, \cdots, \tilde{x}_{M}^{i}\right]^{\top}$. Let $\tilde{y}_{n}$ be the vector concatenation of $\tilde{x}_{n}$ and $\lambda_{n}$. This lemma is proved in two steps. First, it is shown that the conditional differential entropy of $y_{n}$ given $\mathscr{D}_{k}$ is equal to that of $\tilde{y}_{n-1}$ given $\mathscr{D}_{k}$ (see (35)). Next, a relation between the conditional differential entropy of $\tilde{y}_{n-1}$ given $\mathscr{D}_{k}$ and that of $y_{n-1}$ given $\mathscr{D}_{k}$ is established. Note that, $\mathrm{h}\left[y_{n} \mid \mathscr{D}_{k-1}\right]$ can be written as

$$
\begin{aligned}
\mathrm{h}\left[y_{n} \mid \mathscr{D}_{k-1}\right] & =\mathrm{h}\left[x_{n}, \lambda_{n} \mid \mathscr{D}_{k-1}\right] \\
& \stackrel{*}{=} \mathrm{h}\left[\tilde{x}_{n-1}, \lambda_{n-1} \mid \mathscr{D}_{k-1}\right] \\
& =\mathrm{h}\left[\tilde{y}_{n-1} \mid \mathscr{D}_{k-1}\right]
\end{aligned}
$$

where $(*)$ follows from the translation invariance property of the differential entropy and the fact that $Q_{k-1}$ is fixed given $\mathscr{D}_{k-1}=\left\{\hat{Q}_{n}=\hat{q}_{n}\right\}_{n=0}^{k-1}$. Next, we derive an expression for the probability density function (PDF) of $\tilde{y}_{n}$ in terms of the PDF of $y_{n}$. Let $p_{\tilde{y}_{n}}\left(y \mid \mathscr{D}_{k-1}\right)$ and $p_{y_{n}}\left(y \mid \mathscr{D}_{k-1}\right)$ to denote the PDFs of $\tilde{y}_{n}$ and $y_{n}$, respectively, conditioned on $\mathscr{D}_{k-1}$. Let $F(\cdot)$ represent the mapping between $\tilde{y}_{n}$ and $y_{n}$, i.e., $\tilde{y}_{n}=F\left(y_{n}\right)$. Note that $0<1+\mu_{n} \frac{d^{2}}{d x^{i}} U_{i}\left(x^{i}\right)<1$ since $0<\mu_{n}<\min _{i} \frac{1}{\left|U_{i}^{\min }\right|}$ which implies that the mapping $F(\cdot)$ is invertible. Thus, the change-of-variables formula for invertible diffeomorphisms of random vectors (see e.g., (4.63) in [18]) can be applied to write

$$
p_{\tilde{y}_{n-1}}\left(y \mid \mathscr{D}_{k-1}\right)=\frac{1}{\operatorname{det} J_{F}\left[F^{-1}(y)\right]} p_{y_{n-1}}\left(F^{-1}(y) \mid \mathscr{D}_{k-1}\right)
$$

where $J_{F}[x]$ is Jacobian of $F(x)$ evaluated at $x$. Using (36), the conditional entropy of $\tilde{y}_{n-1}$ given $\mathscr{D}_{k-1}$ can be written as

$$
\begin{aligned}
\mathrm{h}\left[\tilde{y}_{n-1} \mid \mathscr{D}_{k-1}\right] & =\int \log \left(\operatorname{det} J_{F}\left[F^{-1}(y)\right]\right) \frac{1}{\operatorname{det} J_{F}\left[F^{-1}(y)\right]} p_{y_{n-1}}\left(F^{-1}(y) \mid \mathscr{D}_{k-1}\right) d y \\
& -\int \log \left(p_{y_{n-1}}\left(F^{-1}(y) \mid \mathscr{D}_{k-1}\right)\right) \frac{1}{\operatorname{det} J_{F}\left[F^{-1}(y)\right]} p_{y_{n-1}}\left(F^{-1}(y) \mid \mathscr{D}_{k-1}\right) d y, \\
& \stackrel{(*)}{=} \int \log \left(\operatorname{det} J_{F}[z]\right) p_{y_{n-1}}\left(z \mid \mathscr{D}_{k-1}\right) d z-\int \log \left(p_{y_{n-1}}\left(z \mid \mathscr{D}_{k-1}\right)\right) p_{y_{n-1}}\left(z \mid \mathscr{D}_{k-1}\right) d z, \\
& =\sum_{j=1}^{M} \mathrm{E}\left[\log \left(1+\mu_{n-1} \frac{d^{2}}{d x^{j}} U_{j}\left(x_{n-1}^{j}\right)\right) \mid \mathscr{D}_{k-1}\right]+\mathrm{h}\left[y_{n-1} \mid \mathscr{D}_{k-1}\right],
\end{aligned}
$$

where $(*)$ follows from the change of variable $z=F^{-1}(x)$.

Using Lemma $1, \mathrm{~h}\left[y_{k} \mid \mathscr{D}_{k-1}\right]$ can be further expanded as 


$$
\mathrm{h}\left[y_{k} \mid \mathscr{D}_{k-1}\right]=\mathrm{h}\left[y_{0} \mid \mathscr{D}_{k-1}\right]+\sum_{j=1}^{M} \sum_{n=0}^{k-1} \mathrm{E}\left[\log \left(1+\mu_{n} \frac{d^{2}}{d x^{j^{2}}} U_{j}\left(x_{n}^{j}\right)\right) \mid \mathscr{D}_{k-1}\right]
$$

Using (38), E $\left[\mathrm{h}\left[y_{k} \mid \mathscr{D}_{k-1}\right]\right]$ can be written as

$$
\mathrm{E}\left[\mathrm{h}\left[y_{k} \mid \mathscr{D}_{k-1}\right]\right]=\sum_{j=1}^{M} \sum_{n=0}^{k-1} \mathrm{E}\left[\log \left(1+\mu_{n} \frac{d^{2}}{d x^{j}} U_{j}\left(x_{n}^{j}\right)\right)\right]+\mathrm{E}\left[\mathrm{h}\left[y_{0} \mid \mathscr{D}_{k-1}\right]\right]
$$

The following lemma, adapted from [1], establishes a lower bound on $\mathrm{E}\left[\mathrm{h}\left[y_{k} \mid \mathscr{D}_{k-1}\right]\right]$ :

Lemma 2. The average conditional entropy of $y_{0}$ given $\mathscr{D}_{k-1}$, i.e., $\mathrm{E}\left[\mathrm{h}\left[y_{0} \mid \mathscr{D}_{k-1}\right]\right]$, can be lower bounded as

$$
\mathrm{E}\left[\mathrm{h}\left[y_{0} \mid \mathscr{D}_{k-1}\right]\right] \geq \mathrm{h}\left[y_{0}\right]-\sum_{t=0}^{k-1}\left(\left(\sum_{i=1}^{M} \log \left|\mathscr{A}_{i, t}^{x}\right|\right)+\sum_{j=1}^{N} \log \left|\mathscr{A}_{j, t}^{\lambda}\right|\right) .
$$

Proof. Follows directly from the first inequality in appendix C in [1]; alternatively, it can be derived from (8.48) and (8.89) in [17].

Applying Lemma 2 to (39) yields

$$
\mathrm{E}\left[\mathrm{h}\left[y_{k} \mid \mathscr{D}_{k-1}\right]\right] \geq \sum_{j=1}^{M} \sum_{n=0}^{k-1} \mathrm{E}\left[\log \left(1+\mu_{n} \frac{d^{2}}{d x^{j^{2}}} U_{j}\left(x_{n}^{j}\right)\right)\right]+\mathrm{h}\left[y_{0}\right]-\sum_{t=0}^{k-1}\left(\left(\sum_{i=1}^{M} \log \left|\mathscr{A}_{i, t}^{x}\right|\right)+\sum_{j=1}^{N} \log \left|\mathscr{A}_{j, t}^{\lambda}\right|\right)
$$

Since $x_{0}$ and $\lambda_{0}$ are independent, the differential entropy of $y_{0}$ can be written as $\mathrm{h}\left[y_{0}\right]=\mathrm{h}\left[x_{0}\right]+\mathrm{h}\left[\lambda_{0}\right]$ which implies that $y_{0}$ has finite differential entropy. Using (32), (33), (40) and the fact that $y_{0}$ has a finite entropy, the DDE can be lower bounded as

$$
\liminf _{k \rightarrow \infty} \frac{1}{k} \log \mathrm{E}\left[\left\|\varepsilon_{k}\right\|_{2}^{2}\right] \geq \frac{2}{M+N}\left(\liminf _{k \rightarrow \infty} \sum_{j=1}^{M} \frac{1}{k} \sum_{n=0}^{k-1} \mathrm{E}\left[\log \left(1+\mu_{n} \frac{d^{2}}{d x^{j^{2}}} U_{j}\left(x_{n}^{j}\right)\right)\right]-R_{\mathscr{Q}}\right) .
$$

The next lemma presents the asymptotic behavior of the first term in the right hand side of equation (41).

Lemma 3. [10] Consider the primal-dual update rule (6) under an OA quantization scheme. Then,

$$
\lim _{k \rightarrow \infty} \sum_{j=1}^{M} \frac{1}{k} \sum_{n=0}^{k-1} \mathrm{E}\left[\log \left(1+\mu_{n} \frac{d^{2}}{d x^{j^{2}}} U_{j}\left(x_{n}^{j}\right)\right)\right]=\sum_{j=1}^{M} \log \left(1+\mu^{\star} \frac{d^{2}}{d x^{j^{2}}} U_{j}\left(x^{j^{\star}}\right)\right) .
$$

Applying Lemma 3 to (41) yields

$$
\liminf _{k \rightarrow \infty} \frac{1}{k} \log \mathrm{E}\left[\left\|\varepsilon_{k}\right\|_{2}^{2}\right] \geq \frac{2}{N+M}\left(\sum_{i=1}^{m} \log \left(1+\mu^{\star} \frac{d^{2}}{d x^{i^{2}}} U_{i}\left(x^{i^{\star}}\right)\right)-R_{\mathscr{Q}}\right) .
$$

which completes the proof. 


\section{References}

1. G. N. Nair and R. J. Evans, "Stabilizability of Stochastic Linear Systems with Finite Feedback Data Rates," SIAM Journal on Control and Optimization, vol. 43, no. 2, pp. 413-436, 2004.

2. G. N. Nair, F. Fagnani, S. Zampieri, and R. J. Evans, "Feedback Control Under Data Rate Constraints: An Overview," Proceedings of the IEEE, vol. 95, no. 1, pp. 108-137, Jan 2007.

3. E. Nekouei, T. Alpcan, G. Nair, and R. J. Evans, "Convergence Analysis of Quantized Primal-dual Algorithms in Network Utility Maximization Problems," IEEE Transactions on Control of Network Systems, vol. PP, no. 99, pp. 1-1, 2016.

4. E. Nekouei, G. N. Nair, and T. Alpcan, "Performance Analysis of Gradient-Based Nash Seeking Algorithms Under Quantization," IEEE Transactions on Automatic Control, vol. 61, no. 12, pp. 3771-3783, Dec 2016.

5. F. Kelly, A. Maulloo, and D. Tan, "Rate control in communication networks: shadow prices, proportional fairness and stability," in Journal of the Operational Research Society, vol. 49, 1998.

6. S. Shakkottai and R. Srikant, "Network Optimization and Control," Found. Trends Netw., vol. 2, no. 3, pp. 271-379, Jan 2007.

7. A. Nedić, A. Olshevsky, A. Ozdaglar, and J. N. Tsitsiklis, "Distributed subgradient methods and quantization effects," in 47th IEEE Conference on Decision and Control (CDC), Dec 2008, pp. 4177-4184.

8. P. Yi and Y. Hong, "Quantized Subgradient Algorithm and Data-Rate Analysis for Distributed Optimization," IEEE Transactions on Control of Network Systems, vol. 1, no. 4, pp. 380-392, Dec 2014.

9. J. S. Freudenberg, R. H. Middleton, and V. Solo, "Stabilization and Disturbance Attenuation Over a Gaussian Communication Channel," IEEE Transactions on Automatic Control, vol. 55, no. 3, pp. 795-799, March 2010.

10. E. Nekouei, T. Alpcan, G. Nair, and R. J. Evans, "Convergence Analysis of Quantized Primal-dual Algorithm in Network Utility Maximization Problems," http://arxiv.org/abs/1604.00723, Tech. Rep., Apr 2016.

11. C. U. Saraydar, N. B. Mandayam, and D. J. Goodman, "Efficient power control via pricing in wireless data networks," IEEE Transactions on Communications, vol. 50, no. 2, pp. 291-303, Feb 2002.

12. J. R. Marden and J. S. Shamma, "Chapter 16 - Game Theory and Distributed Control," ser. Handbook of Game Theory with Economic Applications, H. P. Young and S. Zamir, Eds. Elsevier, 2015, vol. 4, pp. 861-899.

13. N. D. Stein, "Characterization and Computation of Equilibria in Infinite Games," Master's thesis, M.I.T., June 2007.

14. S. Li and T. Basar, "Distributed algorithms for the computation of noncooperative equilibria," Automatica, vol. 23, no. 4, pp. 523-533, 1987.

15. J. B. Rosen, "Existence and Uniqueness of Equilibrium Points for Concave N-Person Games," Econometrica, vol. 33, no. 3, pp. 520-534, 1965.

16. E. Nekouei, T. Alpcan, and D. Chattopadhyay, "Game-Theoretic Frameworks for Demand Response in Electricity Markets," IEEE Transactions on Smart Grid, vol. 6, no. 2, pp. 748-758, March 2015.

17. T. M. Cover and J. A. Thomas, Elements of Information Theory. Wiley-Interscience, 2006.

18. A. Leon-Garcia, Probability, Statistics, and Random Processes for Electrical Engineering, 2nd ed. Massachusetts: Addison-Wesley, 1994. 


\section{University Library}

\section{- M M N E R VA A gateway to Melbourne's research publications}

Minerva Access is the Institutional Repository of The University of Melbourne

Author/s:

Nekouei, E;Alpcan, T;Evans, RJ

Title:

Impact of quantized inter-agent communications on game-theoretic and distributed optimization algorithms

Date:

2018-01-01

Citation:

Nekouei, E., Alpcan, T. \& Evans, R. J. (2018). Impact of quantized inter-agent communications on game-theoretic and distributed optimization algorithms. Ba\#tar, T (Ed.) Uncertainty in Complex Networked Systems, Uncertainty in Complex Networked Systems, (1), pp.501-532. Springer.

Persistent Link:

http://hdl.handle.net/11343/241553 